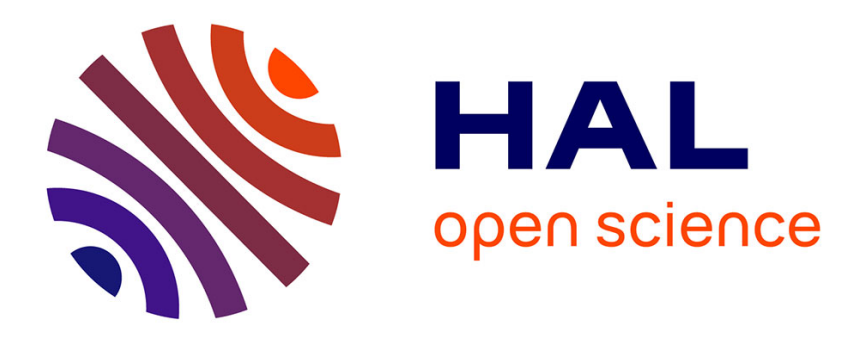

\title{
Analyse micromécanique de la rupture des composites céramiques
}

P. Pérès, L. Anquez, J. Jamet

\section{To cite this version:}

P. Pérès, L. Anquez, J. Jamet. Analyse micromécanique de la rupture des composites céramiques. Revue de Physique Appliquée, 1988, 23 (3), pp.213-228. 10.1051/rphysap:01988002303021300 . jpa00245764

\section{HAL Id: jpa-00245764 https://hal.science/jpa-00245764}

Submitted on 1 Jan 1988

HAL is a multi-disciplinary open access archive for the deposit and dissemination of scientific research documents, whether they are published or not. The documents may come from teaching and research institutions in France or abroad, or from public or private research centers.
L'archive ouverte pluridisciplinaire HAL, est destinée au dépôt et à la diffusion de documents scientifiques de niveau recherche, publiés ou non, émanant des établissements d'enseignement et de recherche français ou étrangers, des laboratoires publics ou privés. 
Classification

Physics Abstracts

$81.20 \mathrm{~N}-46.30 \mathrm{~N}-46.30 \mathrm{P}$

\title{
Analyse micromécanique de la rupture des composites céramiques
}

\author{
P. Pérès, L. Anquez et J. Jamet \\ ONERA, B.P. 72, 92322 Châtillon-sous-Bagneux, France
}

(Reçu le 19 juin 1987, accepté le 11 décembre 1987)

\begin{abstract}
Résumé. - L'analyse mécanique du comportement à la rupture d'un composite céramique/céramique élémentaire constitué d'une fibre gainée de matrice s'appuie sur deux hypothèses essentielles : d'une part l'adaptation locale par glissement fibre-matrice à l'apparition d'une fissure matricielle, et d'autre part la distribution statistique de défauts. Les conséquences de ces hypothèses sur la multifissuration matricielle, sur les comportements non linéaires des composites monofilamentaires sont discutées au regard des résultats expérimentaux sur composites unidirectionnels multifilamentaires $\mathrm{SiC} /$ alumine.
\end{abstract}

\begin{abstract}
The fracture mechanic analysis of a basic ceramic-ceramic composite (filament + coating) is based on two main assumptions : local adaptation by sliding between fibre and matrix when a crack in the matrix appears, and statistical distribution of defects. The consequences of such assumptions concerning the multicracking of the matrix explain the non linear behaviour of such composites. They are discussed in relation with experimental results obtained on unidirectional multifilaments $\mathrm{SiC} /$ alumina composites.
\end{abstract}

\section{Introduction.}

L'ensemble des analyses de la rupture des composites céramiques actuellement proposées $[1,2]$ concerne la propagation de fissures matricielles longues préexistantes pontées par des fibres.

Les premières observations expérimentales menées à l'Office, notamment sur le refroidissement de composites unidirectionnels $\mathrm{SiC} / \mathrm{Al}_{2} \mathrm{O}_{3}$, montrent qu'avant toute création de fissures longues, il y a développement d'une phase d'initiation de ces fissures.

Ainsi, pour de tels systèmes unidirectionnels, nous proposons une première analyse mécanique du comportement à rupture d'une fibre unitaire gainée par une matrice céramique. Elle concerne :

- les distributions de contraintes dans les deux composants en présence d'une fissure matricielle ;

- l'évolution de la microfissuration matricielle en introduisant les notions de défauts et de statistique de rupture ;

- les évolutions des distributions de contraintes au sein des composites microfissurés et l'énergie de frottement dissipée à l'interface lors du cyclage de tels systèmes.

La deuxième partie s'appuie sur des observations REVUE DE PHYSIQUE APPLIQUÉE. - T. 23, N`3, MARS 1988 expérimentales réalisées sur le système composite unidirectionnel multifilamentaire $\mathrm{SiC}$ Nicalon/alumine. Elle tentera de dégager les premières analogies avec les modèles précédemment décrits.

\section{Modèle mécanique du composite monofilamentaire sollicité en traction pure.}

Faisant l'hypothèse que les contraintes dans la direction de l'effort de traction sont prédominantes et que la liaison entre la fibre et la matrice à l'interface se fait par cisaillement, le comportement mécanique de ce composite est défini par un système de deux équations d'équilibre aux dérivées partielles couplées (Fig. 1).

$\mathrm{Ne}$ tenant pas compte de l'équation d'équilibre suivant la direction perpendiculaire à l'axe de traction, ce modèle néglige les effets de Poisson, excluant ainsi les contraintes de frettage $\left(\sigma_{\mathrm{rr}}^{\mathrm{m}}\right)$. Les conditions aux limites du type déplacement ou contrainte sont explicitées dans la figure 2 , compte tenu des équations d'équilibre utilisées.

Les constituants sont supposés homogènes et isotropes.

La résolution numérique des équations d'équilibre a été conduite par un schéma aux différences finies 

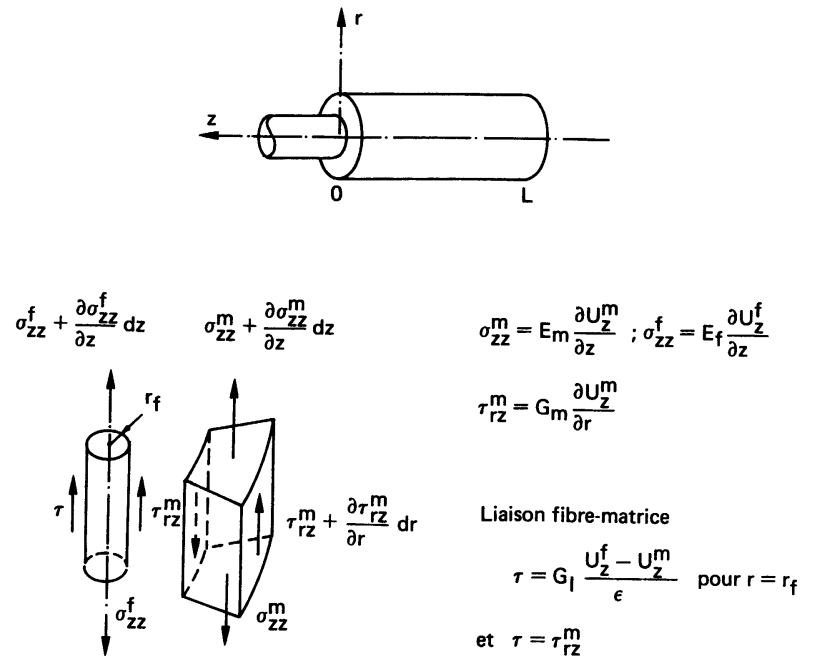

$$
\left\{\begin{array}{l}
E_{f} \frac{\partial^{2} U_{z}^{f}}{\partial z^{2}}+\frac{2 \times \tau \mid r=r_{f}}{r_{f}}=0 \\
E_{m} \frac{\partial^{2} U_{z}^{m}}{\partial z^{2}}+G_{m} \frac{\partial U_{z}^{m}}{\partial r^{2}}+\frac{G_{m}}{r} \frac{\partial U_{z}^{m}}{\partial r}=0
\end{array}\right.
$$

Fig. 1. - Modélisation et équation d'équilibre d'un composite monofilamentaire soumis à un effort de traction.

[Mechanical modelisation and equilibrium equation of a composite in tension.]

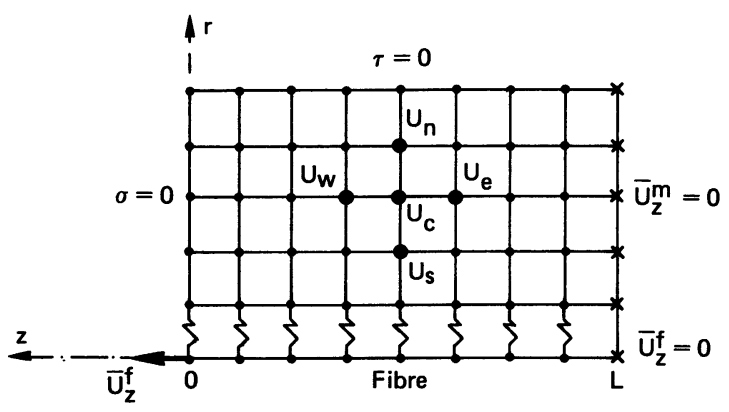

Fig. 2. - Discrétisation par schéma aux différences finies du système composite élémentaire.

[Basic composite discretisation by finite differences method.]

(méthode à cinq points) sur le système discrétisé (Fig. 2).

Les déplacements notés $u$ dans la direction $z$, inconnues du problème, vérifient le système d'équations linéaires suivant :

$$
[K] \cdot\{u\}=\{\bar{u}\}
$$

avec

$[K]$ matrice de « rigidité » (sans dimension); matrice pentadiagonale tenant compte des conditions aux limites du problème des caractéristiques dimensionnelles et de l'élasticité des matériaux

$\{u\}$ vecteur des déplacements de la fibre et de la matrice suivant l'axe $z$

$\{\bar{u}\}$ vecteur des conditions initiales et aux limites.

\section{Analyse des singularités de contrainte à l'interface et introduction de la notion de seuil de glissement.}

3.1 CAS D'UNE LIAISON RIGIDE À L'INTERFACE. Compte tenu des conditions aux limites lors de la création d'une fissure matricielle (Fig. 2) et pour une liaison fibre-matrice forte $\left(G_{I} / \varepsilon=« \infty »\right)$, les diagrammes des contraintes dans la matrice révèlent une singularité de contrainte de cisaillement à l'interface (Fig. 3a).

La fissure perturbe très localement le chargement de la matrice qui, à une distance caractéristique de la fissure, atteint une valeur constante (Fig. 3b). La fibre se décharge rapidement avant d'atteindre une valeur constante (Fig. 3c).

Des états de contraintes similaires ont été obtenus par la résolution des équations complètes de l'élasticité, en axisymétrique, par la méthode des éléments finis, qui valident le modèle aux différences finies pour des taux volumiques de fibres habituellement rencontrés dans les composites céramiques $(20<$ $V_{\mathrm{f}}<50 \%$ ) [3]. Cette notion de singularité de contrainte n'ayant pas de signification physique, il faut admettre qu'il y ait adaptation locale du matériau. Dans le cas présent, où les deux composants sont fragiles, cette adaptation a été figurée par un glissement relatif entre la fibre et la matrice intervenant au-delà d'un seuil de glissement que nous avons a priori considéré constant et noté $\tau_{1}$. La figure 4 , qui schématise cette rhéologie de l'interface, suppose de plus une énergie de décohésion nulle à l'initiation du glissement interfacial.

3.2 INTRODUCTION DE LA NOTION DE SEUIL DE GLISSEMENT DANS LA MODÉLISATION. - La résolution de ce problème a nécessité le développement d'un algorithme de calcul basé sur un processus itératif pour chaque incrément de chargement du composite.

En supposant les déformations et les déplacements petits, le système d'équations (1) peut être linéarisé, tel que pour un incrément de charge :

$$
[K] \cdot\left\{\delta u_{\mathrm{e}}\right\}=\{\delta \bar{u}\}
$$

où $\delta u_{\mathrm{e}}$ : déplacement élastique.

Soit la fonction $f$ telle que à l'interface on ait :

$$
\text { - } f\left(\tau_{\mathrm{rz}}\right)=\tau_{\mathrm{rz}}^{2}-\tau_{1}^{2}=0
$$

- élasticité pure si $\left|\tau_{\mathrm{rz}}\right|<\tau_{1}$

- glissement interfacial si $\left|\tau_{\mathrm{rz}}\right|>\tau_{1}$ 
Caractéristiques SiC/verre

$$
\begin{aligned}
& E_{f}=206 \mathrm{GPa} \\
& E_{m}=75 \mathrm{GPa} \\
& G_{m}=31,25 \cdot \mathrm{GPa} \\
& G_{I}=" \infty^{\prime \prime} \\
& r_{f}=5.10^{-3} \mathrm{~mm} ; V_{f}=1 / 9 \\
& L=0,5 \mathrm{~mm} \\
& \bar{U}_{f}=1,46.10^{-3} \mathrm{~mm}
\end{aligned}
$$

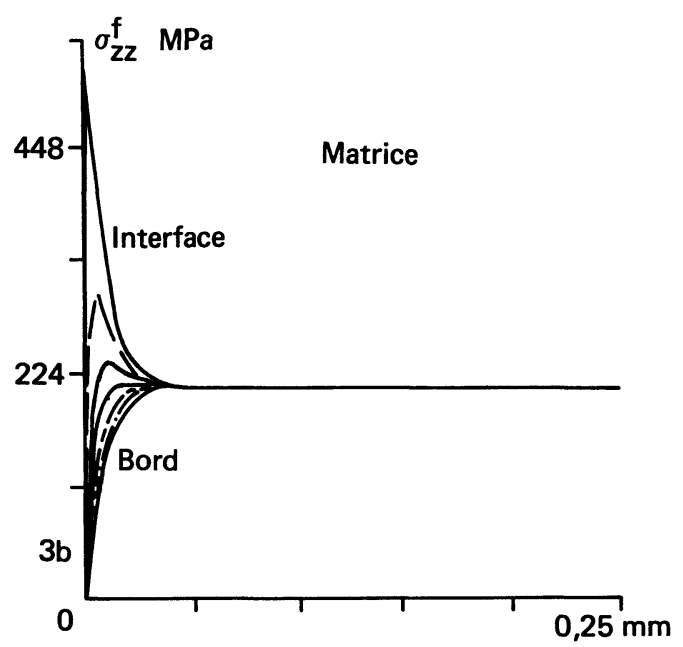

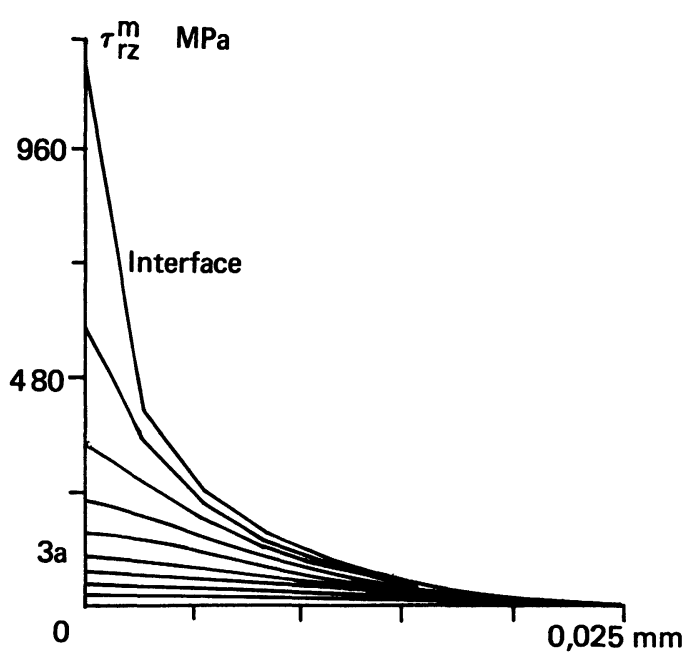

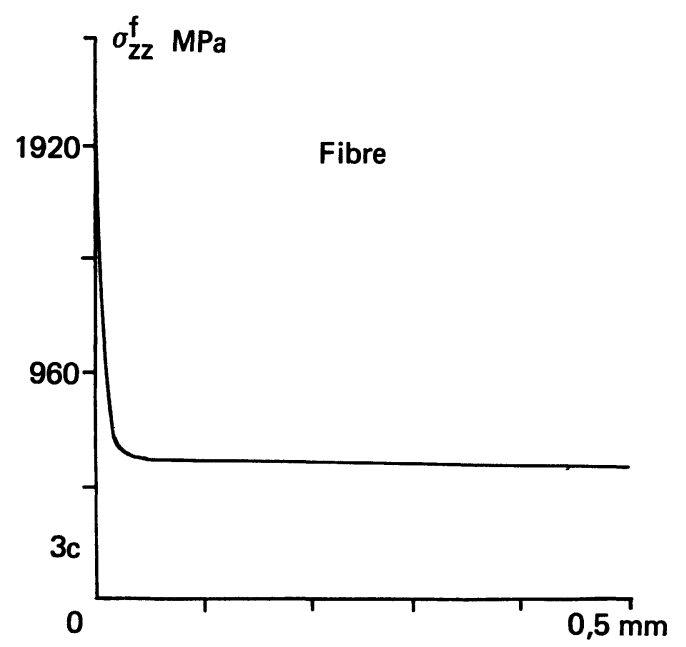

Fig. 3. - Etats de contraintes au voisinage d'une fissure matricielle dans un composite monofilamentaire SiC/verre : a) évolution des contraintes de cisaillement dans la matrice le long de l'axe de traction, b) évolution des contraintes de traction dans la matrice le long de l'axe de traction, c) évolution des contraintes de traction dans la fibre le long de l'axe de traction.

[Stresses state near a crack in a SiC/glass composite : a) shear stresses evolution in the matrix along the tensile axis, b) tensile stresses evolution in the matrix along the tensile axis, c) tensile stresses evolution in the fibre along the tensile axis.]

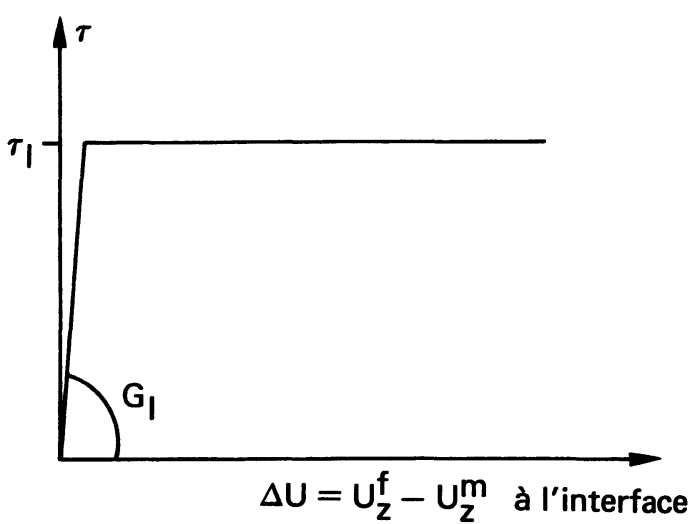

Fig. 4. - Modélisation du comportement de l'interface en cisaillement.

[Interfacial shear behaviour model.] avec $\tau_{\mathrm{rz}}=G_{\mathrm{I}} / \varepsilon \cdot\left(\delta u_{\mathrm{e}}^{\mathrm{f}}-\delta u_{\mathrm{e}}^{\mathrm{m}}\right)=G_{\mathrm{I}} / \varepsilon \cdot \delta u_{\mathrm{e}}^{\mathrm{I}}$

où $\mathbf{f}$ symbolise la fibre m symbolise la matrice

I symbolise l'interface

e symbolise l'élasticité .

Durant le chargement, la zone de frottement à l'interface augmente d'une façon telle que la fonction $f$ vérifie :

$$
f\left(\tau_{\mathrm{rz}}, \tau_{\mathrm{g}}\right)=\left(\tau_{\mathrm{rz}}-\tau_{\mathrm{g}}\right)^{2}-\tau_{1}^{2}=0
$$

où $\tau_{\mathrm{g}}=G_{\mathrm{I}} / \varepsilon \cdot \delta u_{\mathrm{g}}^{\mathrm{I}}$, $\delta u_{\mathrm{g}}^{\mathrm{I}}$ représentant le déplacement relatif de la fibre par rapport à la matrice à l'interface, dû au glissement, pour un incrément de charge. 
L'équation (4) permet d'écrire :

$$
\begin{aligned}
\tau_{\mathrm{rz}}^{2} & =\left[G_{\mathrm{I}} / \varepsilon \cdot\left(\delta u^{\mathrm{I}}-\delta u_{\mathrm{g}}^{\mathrm{I}}\right)\right]^{2}=\tau_{1}^{2} \\
\text { avec } \delta u^{\mathrm{I}} & =\delta u_{\mathrm{e}}^{\mathrm{I}}+\delta u_{\mathrm{g}}^{\mathrm{I}} \\
\delta u^{\mathrm{I}} & =\text { déplacement relatif total fibre-matrice }
\end{aligned}
$$

d'où l'on peut écrire que :

$$
\begin{array}{ll} 
& G_{\mathrm{I}} / \varepsilon \cdot\left(\delta u^{\mathrm{I}}-\delta u_{\mathrm{g}}^{\mathrm{I}}\right)=\tau_{1} \cdot \tau_{\mathrm{rz}} /\left|\tau_{\mathrm{rz}}\right| \\
\text { donc } \quad & \delta u_{\mathrm{g}}^{\mathrm{I}}=-\tau_{1} \cdot \tau_{\mathrm{rz}} /\left|\tau_{\mathrm{rz}}\right| \cdot 1 /\left(G_{\mathrm{I}} / \varepsilon\right)+\delta u^{\mathrm{I}}
\end{array}
$$

$\delta u_{\mathrm{g}}^{\mathrm{I}}$ correspond au nouveau déplacement relatif fibre-matrice à l'interface dû au glissement dans l'incrément de pas de chargement. L'équation (2) s'écrit donc :

$$
[K] \cdot\left\{\delta u-\delta u_{\mathrm{g}}^{\mathrm{I}}\right\}=\{\delta \bar{u}\}
$$

qu'on écrit

$$
[K] \cdot\{\delta u\}=\{\delta \bar{u}\}+\left\{\delta \tilde{u}_{\mathrm{g}}\right\}
$$

avec $\left\{\delta \tilde{u}_{\mathrm{g}}\right\}=$ vecteur des déplacements de frottement, dont les composantes sur la fibre et sur la matrice à l'interface s'écrivent respectivement :

$$
\begin{aligned}
& \delta \tilde{u}_{\mathrm{g}}^{\mathrm{f}}=G_{\mathrm{I}} / \varepsilon \cdot K_{1} /\left(E_{\mathrm{f}} r_{\mathrm{f}}\right) \cdot \delta u_{\mathrm{g}}^{\mathrm{I}} \\
& \delta \tilde{u}_{\mathrm{g}}^{\mathrm{m}}=G_{\mathrm{I}} / \varepsilon \cdot K_{2} / E_{\mathrm{m}} \cdot \delta u_{\mathrm{g}}^{\mathrm{I}}
\end{aligned}
$$

où $K_{1}$ et $K_{2}$ sont des constantes.

L'algorithme de résolution utilisé pour résoudre le problème de glissement à seuil peut être schématisé comme suit :

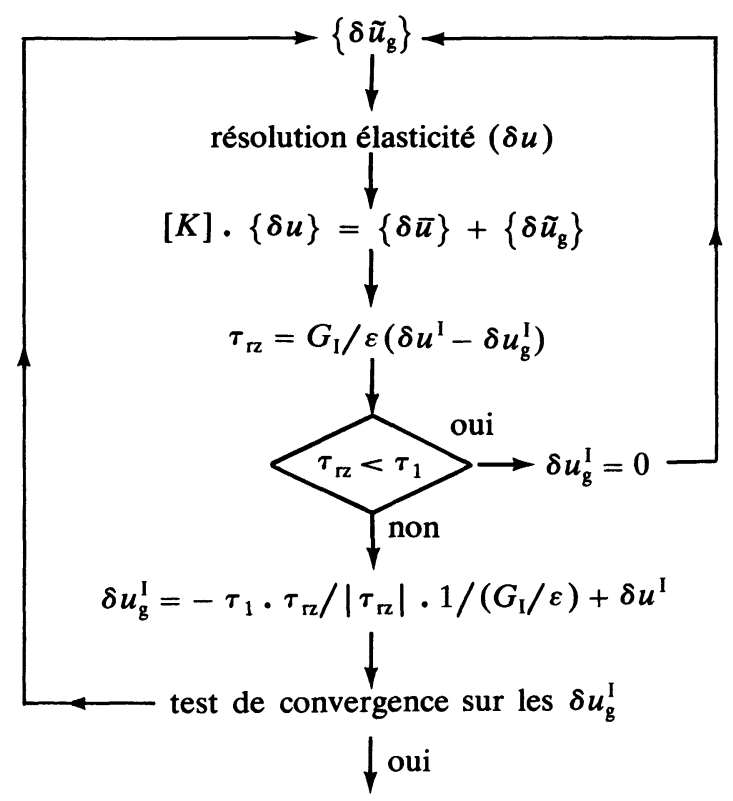

La convergence, en général monotone, de cet algorithme devient lente lorsque la rigidité en cisaillement de l'interface $\left(G_{\mathrm{I}} / \varepsilon\right)$ augmente.

Les états de contraintes dans la fibre et dans la matrice sont représentés sur les figures $5 \mathrm{a}, 5 \mathrm{~b}, 5 \mathrm{c}$, pour lesquelles le seuil de glissement $\tau_{1}$ a été fixé à $10 \mathrm{MPa}$ et dont les caractéristiques des constituants et de chargement sont identiques à l'exemple précédent (cas des composites $\mathrm{SiC} /$ verre). La valeur $G_{\mathrm{I}} / \varepsilon$ a été choisie telle que le rapport relatif déplacement interfacial fibre-matrice en dehors de la zone de glissement soit inférieur à $10^{-3}$.

Ces distributions montrent un déchargement linéaire de la fibre (Fig. 5a) et un chargement linéaire (quasiment sans gradient dans l'épaisseur de matrice) dans la zone de glissement 1 (Fig. 5b). Audelà de cette zone, une surcontrainte de traction de la matrice à l'interface apparaît. Des raffinements successifs du maillage ont montré qu'elle avait une valeur finie significative. Elle est caractéristique des conditions aux limites utilisées à l'interface et de la valeur $G_{\mathrm{I}} / \varepsilon$. Les contraintes de cisaillement dans la matrice (Fig. 5c) présentent un gradient suivant l'épaisseur de matrice dans la zone de glissement. En effet, on peut écrire en première approximation que :

$$
\tau_{\mathrm{rz}}=\left(\tau_{1} \cdot r_{\mathrm{f}}\right) /\left(r_{\mathrm{m}}^{2}-r_{\mathrm{f}}^{2}\right)\left(-r+r_{\mathrm{m}}^{2} / r\right)
$$

$r_{\mathrm{m}}$ : rayon de la matrice ; $r_{\mathrm{f}}$ : rayon de la fibre où $\tau_{\mathrm{rz}}$ vérifie l'équation d'équilibre suivante :

$$
\frac{\partial \sigma_{\mathrm{zz}}}{\partial z}+\frac{1}{r} \frac{\partial}{\partial r}\left(r \cdot \tau_{\mathrm{rz}}\right)=0 .
$$

La longueur de glissement $l$ vérifie l'équation suivante :

$$
l=\left(1-V_{\mathrm{f}}\right) / V_{\mathrm{f}} \cdot\left(\sigma_{\mathrm{m}} \cdot r_{\mathrm{f}}\right) /\left(2 \tau_{1}\right)
$$

avec

$\sigma_{\mathrm{m}} \quad$ : valeur de la contrainte dans la matrice à la distance caractéristique $l$

$V_{\mathrm{f}} \quad$ : taux volumique de fibre $V_{\mathrm{f}}=r_{\mathrm{f}}^{2} / r_{\mathrm{m}}^{2}$.

Cette analyse mécanique basée sur l'hypothèse du glissement interfacial peut rendre compte du phénomène de fissurations multiples au sens d'ACK en supposant l'unicité de la contrainte de rupture de la matrice.

\section{Analyse de la rupture par approche statistique.}

Cependant, cette approche est insuffisante pour décrire un système céramique/céramique, où les défauts peuvent jouer un rôle important. C'est pourquoi nous faisons maintenant l'hypothèse que la rupture fragile de la matrice est gérée par la statistique de Weibull et que la propagation instantanée des fissures autour de la fibre déclenche un glissement interfacial.

Ainsi cette seconde partie, s'appuyant sur les résultats de l'analyse mécanique précédente, introduit maintenant de façon simple la notion de statistique de rupture à la fois de la matrice et de la fibre. 

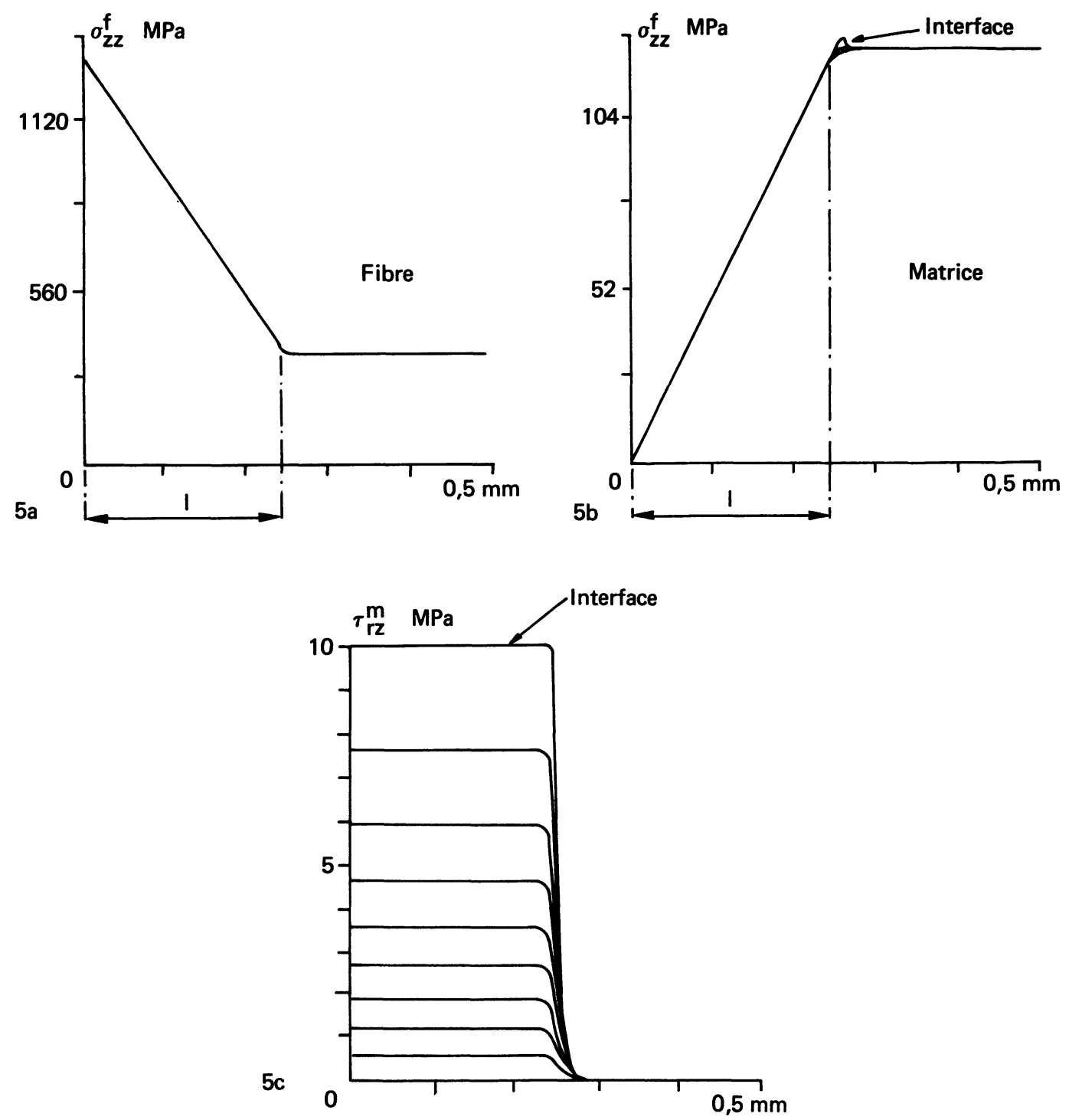

Fig. 5. - Etats de contraintes au voisinage d'une fissure matricielle après glissement interfacial dans un composite monofilamentaire SiC/verre : a) évolution des contraintes de traction dans la fibre le long de l'axe de traction, b) évolution des contraintes de traction dans la matrice le long de l'axe de traction, c) évolution des contraintes de cisaillement dans la matrice le long de l'axe de traction.

[Stresses state along the tensile axis near a crack after interfacial sliding in a SiC/glass composite : a) tensile stresses evolution in the fibre, b) tensile stresses evolution in the matrix, c) shear stresses evolution in the matrix.]

Faisant l'hypothèse de défauts de volume dans la matrice et de défauts linéaires dans la fibre, les calculs analytiques de probabilité dans le composite monofilamentaire ont été menés en fixant arbitrairement la probabilité de rupture des constituants à 0,5 (contrainte médiane de rupture).

La probabilité de rupture de la matrice et de la fibre s'exprime respectivement par :

$$
F\left(\sigma_{\mathrm{m}}\right)=1-\exp \left(-\int_{V}\left(\sigma / \stackrel{\circ}{\mathrm{m}}^{a_{\mathrm{m}}} \mathrm{d} V\right)\right.
$$

$$
F\left(\sigma_{\mathrm{f}}\right)=1-\exp \left(-\int_{2 L}\left(\sigma / \stackrel{\circ}{\sigma}_{\mathrm{f}}\right)^{a_{\mathrm{f}}} S \mathrm{~d} L\right)
$$

où

$a$ : représente le coefficient de Weibull (paramètre de forme)

$\stackrel{\circ}{\sigma}$ : est le coefficient d'échelle lié à un volume de référence pour la matrice et à une longueur de référence pour la fibre.

L'approche utilisée consiste à calculer la contrainte d'apparition d'une fissure située entre deux autres 
fissures existantes distantes d'une longueur $2 L$, pour une probabilité de rupture à 0,5 (Fig. 6a).
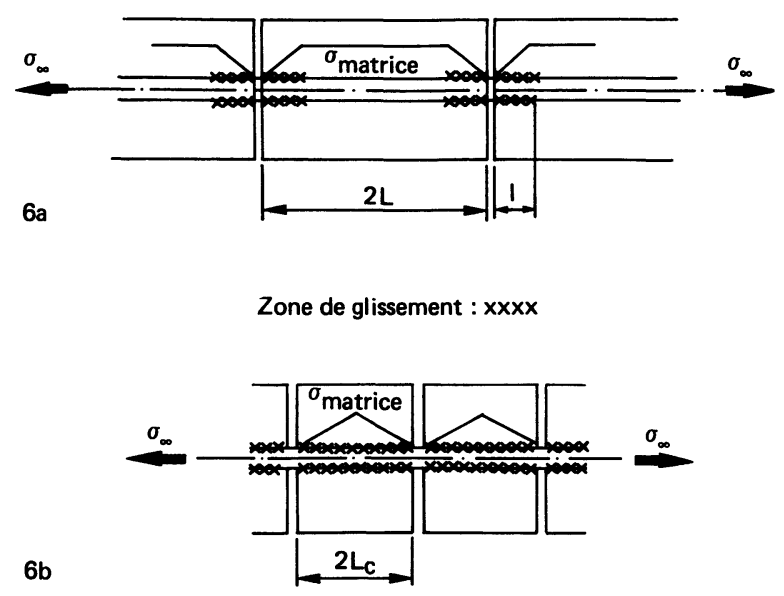

Fig. 6. - Modélisation de la fissuration matricielle dans le composite monofilamentaire : a) fissuration non saturée, b) fissuration saturée $\left(2 L=2 l_{\mathrm{c}}\right)$.

[Modelisation of multicracking in the matrix of composite systems : a) non saturated cracking, b) saturated cracking.]

Cette analyse permet ainsi de calculer en continu :

- la déformation à l'apparition de la première fissure $\left(\varepsilon_{0}\right)$ :

$$
\varepsilon_{0}=\stackrel{\circ}{\sigma}_{\mathrm{m}} / E_{\mathrm{m}}\left((\ln 2) /\left(2 L_{0} \cdot S_{\mathrm{m}}\right)\right)^{1 / a_{\mathrm{m}}}
$$

avec

$S_{\mathrm{m}}$ : surface latérale de la matrice

$E_{\mathrm{m}}$ : module d'Young de la matrice

$2 L_{0}$ : longueur initiale du composite

- la distance interfissure $(2 L)$ en fonction du chargement :

$$
\begin{aligned}
2 L=\frac{\ln 2}{S_{\mathrm{m}}} \cdot\left(\frac{\stackrel{\circ}{\mathrm{m}}_{\mathrm{m}} \cdot V_{\mathrm{m}}(1+1 / \beta)}{\sigma_{\mathrm{c}}}\right)^{a_{\mathrm{m}}}- \\
-2 l\left(\frac{1}{a_{\mathrm{m}}+1}-1\right)
\end{aligned}
$$

avec

$$
\begin{aligned}
\beta=\left(E_{\mathrm{m}} V_{\mathrm{m}}\right) /\left(E_{\mathrm{f}} V_{\mathrm{f}}\right), \\
l=\sigma_{\mathrm{c}} /(1+1 / \beta) \cdot 1 / V_{\mathrm{f}} \cdot r_{\mathrm{f}} /\left(2 \tau_{1}\right)
\end{aligned}
$$

$\sigma_{\mathrm{c}}=F / S(S:$ section du composite, $F:$ force appliquée)

- la déformation moyenne $\left(\bar{\varepsilon}_{\mathrm{f}}\right)$ du composite en fonction du chargement :

- avant la saturation de la fissuration $\left(l_{\mathrm{c}}\right)$ (Fig. 6b)

$$
\bar{\varepsilon}_{\mathrm{f}}=\sigma_{\mathrm{c}} / E_{\mathrm{c}}\left[1+l /(2 L) \cdot E_{\mathrm{m}} / E_{\mathrm{f}} \cdot\left(1-V_{\mathrm{f}}\right) / V_{\mathrm{f}}\right]
$$

$E_{\mathrm{f}}$ : module d'Young de la fibre

$E_{\mathrm{c}}$ : module d'élasticité du composite $\left(E_{\mathrm{c}}=\right.$ $\left.E_{\mathrm{f}} V_{\mathrm{f}}+E_{\mathrm{m}} V_{\mathrm{m}}\right)$

- après la saturation de la fissuration

$$
\bar{\varepsilon}_{\mathrm{f}}=\frac{1}{E_{\mathrm{f}} V_{\mathrm{f}}} \cdot \sigma_{\mathrm{c}}-\frac{l_{\mathrm{c}} \tau_{1}}{r_{\mathrm{f}} E_{\mathrm{f}}}
$$

- le chargement lors de la rupture du renfort au droit de la fissure (cas avant la saturation de la fissuration) :

$$
\sigma_{\mathrm{c}}=(1+\beta) V_{\mathrm{f}} \cdot \stackrel{\circ}{\sigma}_{\mathrm{f}}\left(\ln 2 /\left(\pi \cdot r_{\mathrm{f}}^{2} \cdot 2 L_{0}\right) \cdot 1 / B\right)^{1 / a_{\mathrm{f}}}
$$

avec

$$
\begin{aligned}
B=l / L \cdot 1 /\left(a_{\mathrm{f}}+1\right) \cdot 1 / \beta\left[(1+\beta)^{a_{\mathrm{f}}+1}-1\right]+ & + \\
+ & (1-l / L) .
\end{aligned}
$$

Le détail des calculs est explicité en annexe A.

L'algorithme (Fig. 7) permet de simuler le comportement de composites monofilamentaires lors de sollicitations de traction à forces imposées.

Il a été appliqué à deux systèmes composites céramiques connus : $\mathrm{SiC} /$ verre et $\mathrm{SiC} / \mathrm{SiC}$. Dans le premier cas, la contrainte $\tau_{1}$ est maintenue constante. Le diagramme $\sigma / \varepsilon$ (Fig. 8a) montre que l'apparition de la première fissure dépend du taux volumique de fibre, révélant ainsi l'effet du volume sur l'allongement à rupture de la matrice dans le composite. Dans le deuxième cas, le taux volumique de fibre $V_{\mathrm{f}}$ a été maintenu constant et le diagramme $\sigma / \varepsilon$ (contrainte moyenne composite/déformation moyenne composite: $\bar{\varepsilon}_{\mathrm{f}}$ ) (Fig. $8 \mathrm{~b}$ ) montre que l'apparition de la première fissure correspond à une valeur unique de l'allongement, et donc est indépendante de $\tau_{1}$. L'augmentation de $\tau_{1}$ permet une contribution croissante de la matrice dans la rigidité du composite, différant ainsi la rupture de pente des courbes $\sigma / \varepsilon$. Par ailleurs, ces diagrammes indiquent les points de saturation de la microfissuration de la matrice et la rupture du renfort.

Ainsi donc, l'introduction de la notion de statistique dans la mécanique de la rupture d'un tel système met en évidence la possibilité du phénomène de retard à la microfissuration, qui trouve son origine ici dans la notion de volume concerné par les sollicitations. En d'autres termes, toutes choses égales par ailleurs, un accroissement du taux volumique de renforcement correspond à une réduction du volume de matrice concerné par la sollicitation, et par suite à un accroissement de la contrainte de rupture de cette matrice.

Par ailleurs, compte tenu des hypothèses du modèle, il est logique que la contrainte d'apparition de la première fissure soit strictement indépendant de $\tau_{1}$ puisque le glissement est une conséquence de la présence de la fissure à l'interface. 

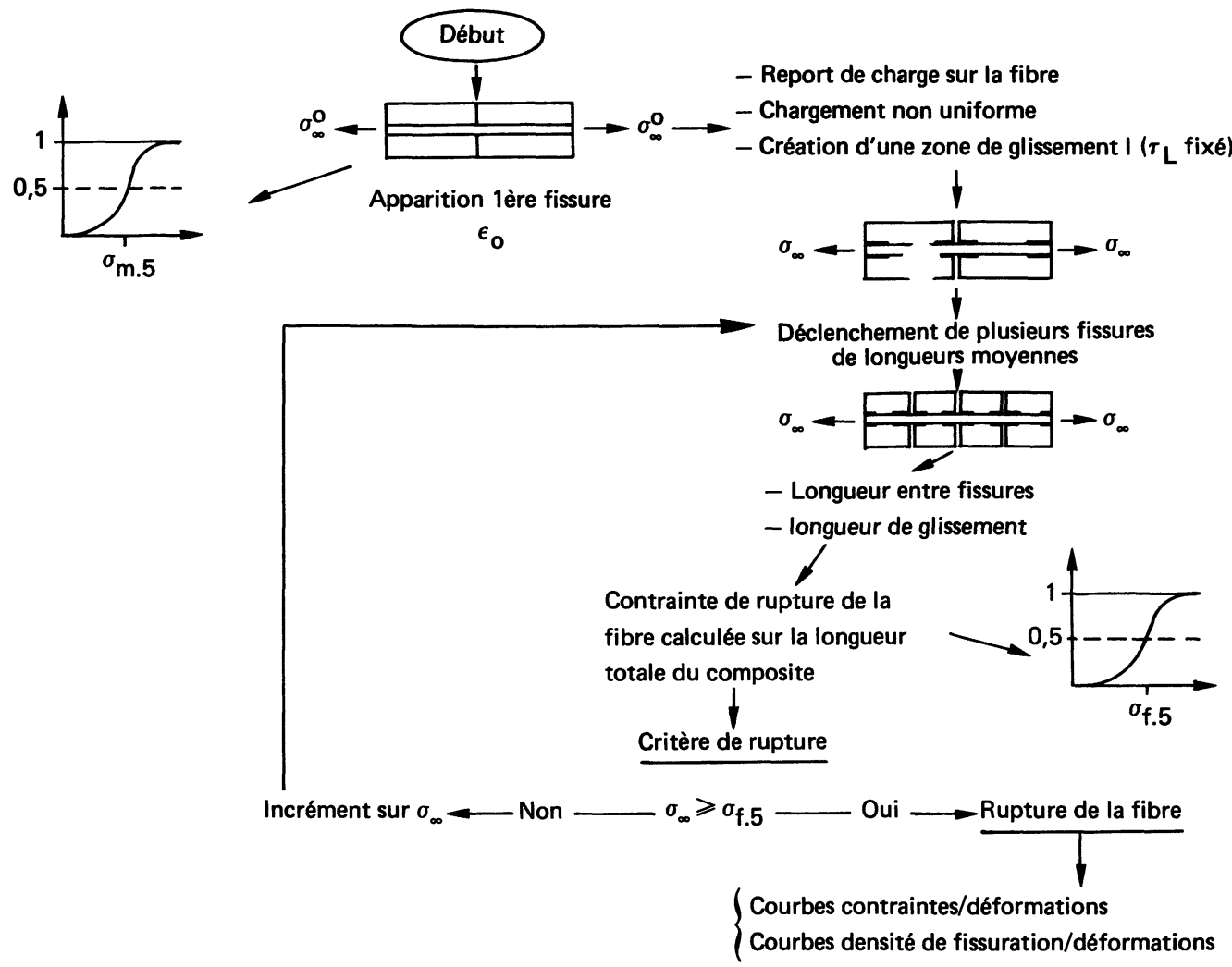

Fig. 7. - Algorithme de calcul de l'évolution de la fissuration matricielle en fonction du chargement. [Analytical expression of the multicracking evolution.]
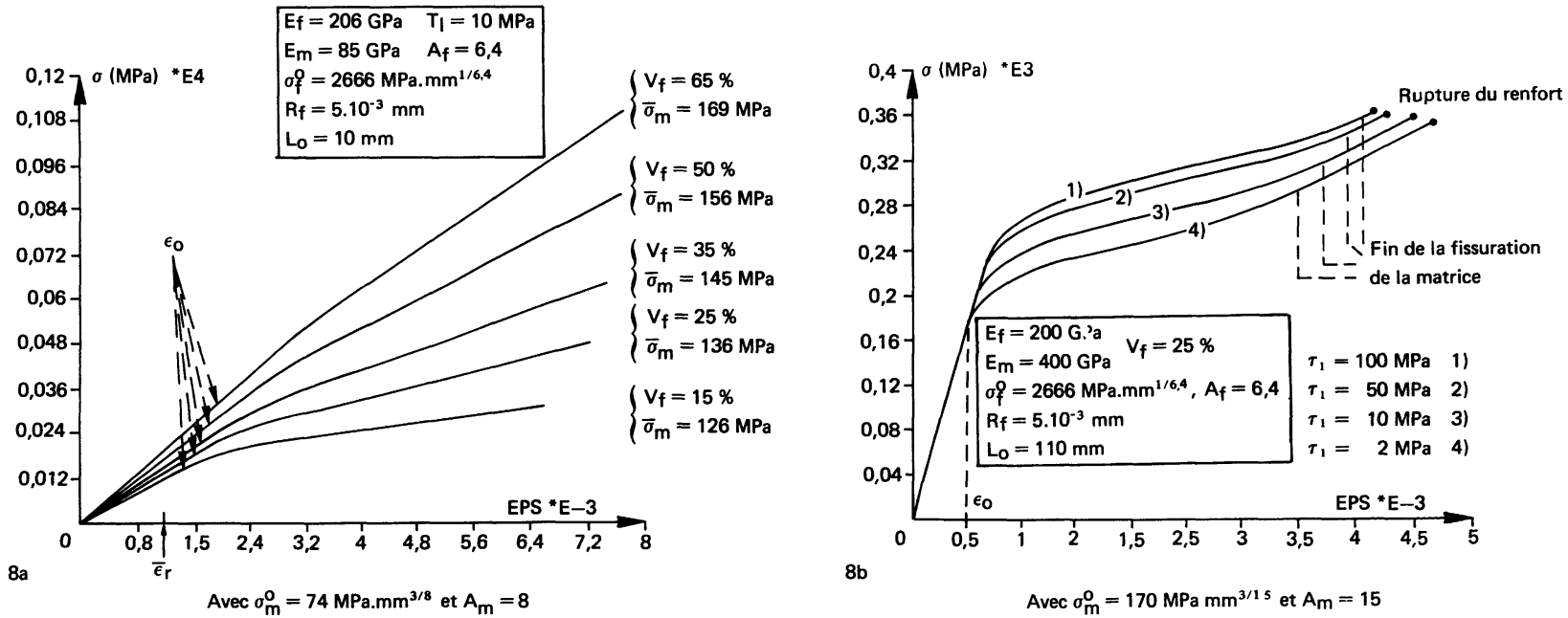

Fig. 8. - Simulation d'essais de traction sur composite céramique monofilamentaire : a) composite SiC/verre. Influence du taux volumique de fibre, $\varepsilon_{0}$ déformation à l'apparition de la $1^{\text {ère }}$ fissure, $\varepsilon_{\mathrm{r}}$ déformation à rupture de la matrice seule, b) composite $\mathrm{SiC} / \mathrm{SiC}$. Influence du seuil de glissement.

[Computed tensile loading curves : a) SiC/glass composite - Effect of the fibre volume fraction, $\varepsilon_{0}$ : first crack in the matrix, $\varepsilon_{\mathrm{r}}$ : single matrix fracture, b) $\mathrm{SiC} / \mathrm{SiC}$ composite - Effect of the limiting interfacial shear stress.] 

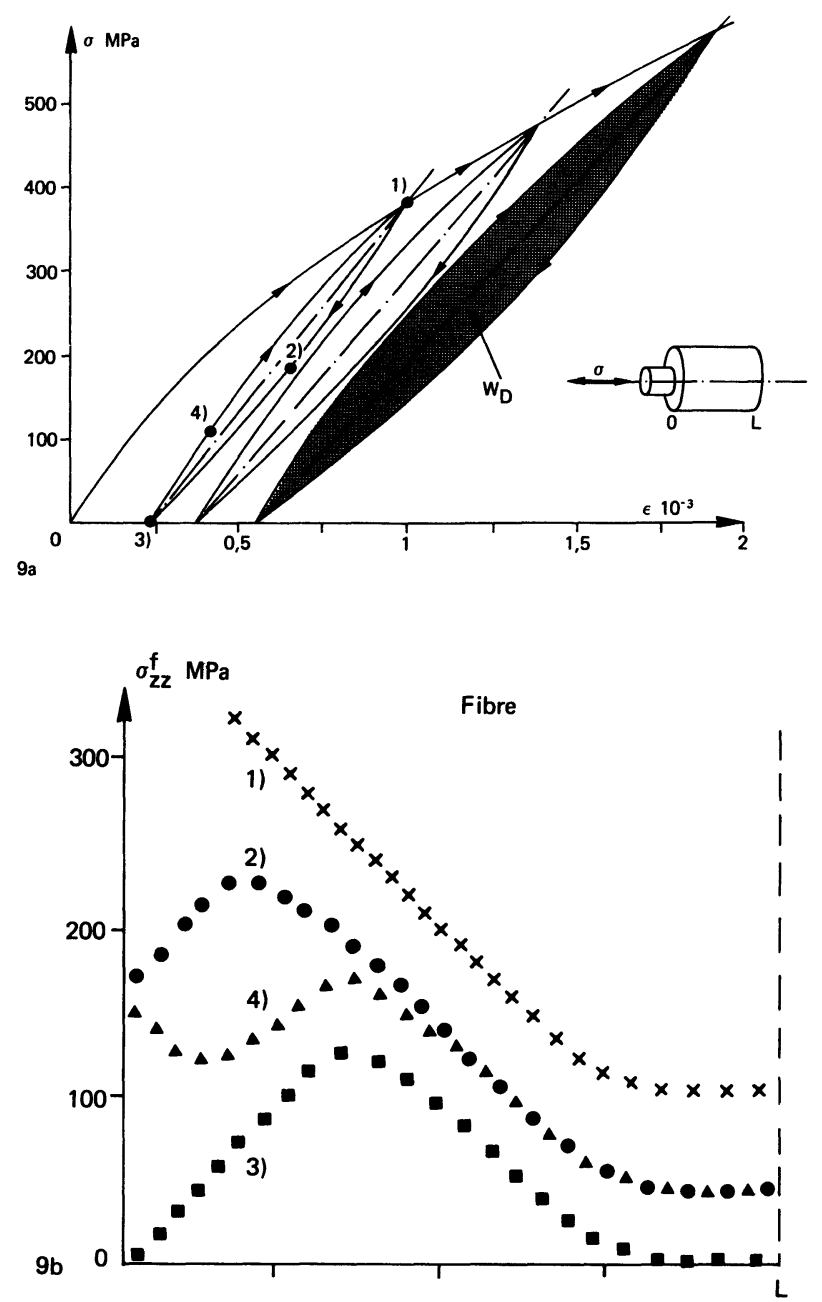
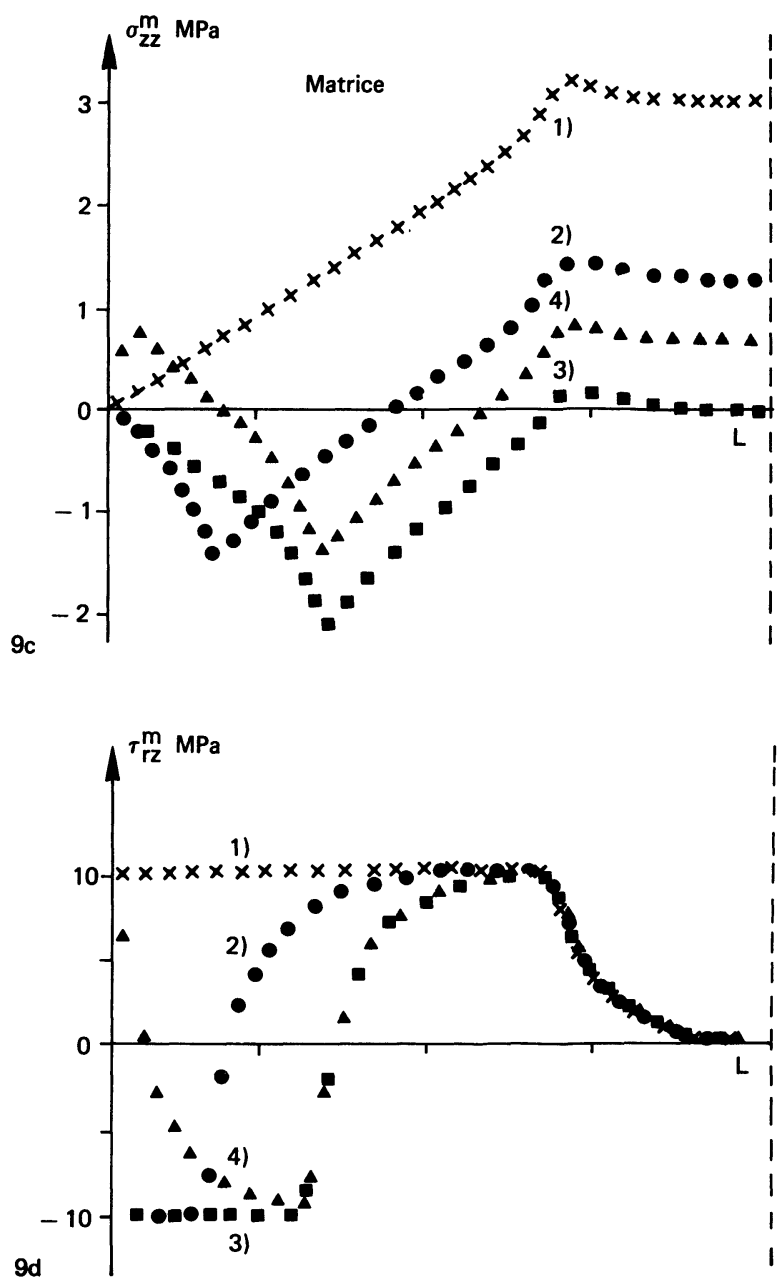

Fig. 9. - Simulation des boucles d'hystérésis lors de chargements cycliques et évolution des états de contraintes dans les composants : a) courbe de cyclage d'un composite SiC/verre monofilamentaire

1) $\varepsilon=0,1 \% \quad \tau_{1}=10 \mathrm{MPa}$

2) $\varepsilon=0,0625 \% \mathrm{~L}=0,1 \mathrm{~mm}$

3) $\varepsilon=0,025 \%$

4) $\varepsilon=0,0375 \%$,

b) évolution des contraintes de traction dans la fibre, c) évolution des contraintes de traction dans la matrice,

d) évolution des contraintes de cisaillement dans la matrice à l'interface.

[Computed unloading/reloading curves : a) SIC/glass composite, b) tensile stresses evolution in the fibre, c) tensile stresses evolution in the matrix, d) shear stresses evolution in the matrix close to the interface.]

Par ailleurs, cette approche statistique et la notion de seuil de glissement permettent de décrire l'évolution des densités de microfissures matricielles en fonction du chargement, et par suite le comportement non linéaire du système jusqu'à la saturation de la microfissuration. Soulignons de plus que la loi de saturation décrite par une distance interfissure moyenne est strictement conforme à celle fournie par la première approche déterministe (Eq. (6)).

Enfin, la rupture du renforcement, elle-même gérée par la statistique, est dépendante de l'état de microfissuration matricielle.

\section{Analyse du comportement non linéaire des systè-} mes microfissures.

Ainsi, après avoir introduit successivement la notion de seuil de glissement à l'interface $\tau_{1}$ et celle de défauts dans les constituants, nous analysons dans le présent chapitre les conséquences de la première notion sur le comportement non linéaire des systèmes monofilamentaires préfissurés. Pour cela, nous avons repris le modèle décrit au chapitre 3 , pour lequel nous avons introduit des chargements cycliques sur l'extrémité du renforcement.

La figure 9a donne un exemple de courbe de cyclage traction-compression du composite monofilamentaire $\mathrm{SiC} /$ verre à partir d'un état pour lequel les contraintes internes sont supposées nulles ( $\sigma$ : contrainte relative à la fibre).

Les figures $9 b, c, d$ illustrent les différents états de contraintes le long de l'axe de traction pour différents allongements moyens de la fibre lors $\mathrm{du}$ chargement et du déchargement pour un seuil de 
glissement fixé à $10 \mathrm{MPa}$. Seul l'état de contrainte matricielle à l'interface a été représenté.

Le comportement en cyclage modélisé ici concerne une seule fissure située à une extrémité du système. Il est représentatif d'un système composite comportant $N$ fissures, avec un rapport de similitude égal à $N$ en supposant $\tau_{1}$ constant dans l'ensemble du composite.

L'énergie de frottement dissipée dans une boucle d'hystérésis (Fig. 9a) ( $\left.W_{\mathrm{D}}\right)$ peut être évaluée en première approximation par l'équation suivante :

$$
\begin{aligned}
W_{\mathrm{D}}=2 & \pi r_{\mathrm{f}} l^{2} \tau_{1} / E_{\mathrm{f}} \times \\
& \times\left[\sigma-11 / 6 E_{\mathrm{c}} /\left(E_{\mathrm{m}} V_{\mathrm{m}}\right) \cdot l / r_{\mathrm{f}} \cdot \tau_{1}\right] .
\end{aligned}
$$

Dans le cas où il n'y a pas glissement sur toute la longueur $L$ l'équation se simplifie, donnant:

$W_{\mathrm{D}}=\pi / 24 \cdot\left(E_{\mathrm{m}} V_{\mathrm{m}} / E_{\mathrm{c}}\right)^{2} \cdot 1 / E_{\mathrm{f}} \cdot r_{\mathrm{f}}^{3} / \tau_{1} \cdot \sigma^{3}$

$\sigma$ : contrainte appliquée à la fibre au début du déchargement.

Ces équations sont explicitées en annexe B.

6. Etude expérimentale du comportement mécanique du composite SiC/alumine unidirectionnel.

6.1 DESCRIPTION DU COMPOSITE $\mathrm{SiC} / \mathrm{Al}_{2} \mathrm{O}_{3}$. Ces composites ont fait l'objet d'une étude de processus d'élaboration à l'ONERA [4].

Ils se caractérisent par l'existence de microfissurations multiples qui se sont développées au cours du refroidissement suivant immédiatement le frittage. Elles ont pour origine les contraintes thermomécaniques générées par le désaccord dilatométrique fibrematrice.

En effet, le coefficient de dilatation de la matrice d'alumine est supérieur à celui de la fibre $\mathrm{SiC}$ Nicalon $\quad\left(\alpha_{\mathrm{m}}=8,7 \times 10^{-6}{ }^{\circ} \mathrm{C}^{-1} ; \quad \alpha_{\mathrm{f}}=3 \times\right.$ $\left.10^{-6}{ }^{\circ} \mathrm{C}^{-1}\right)$.

La figure 10 montre l'état de fissuration, après

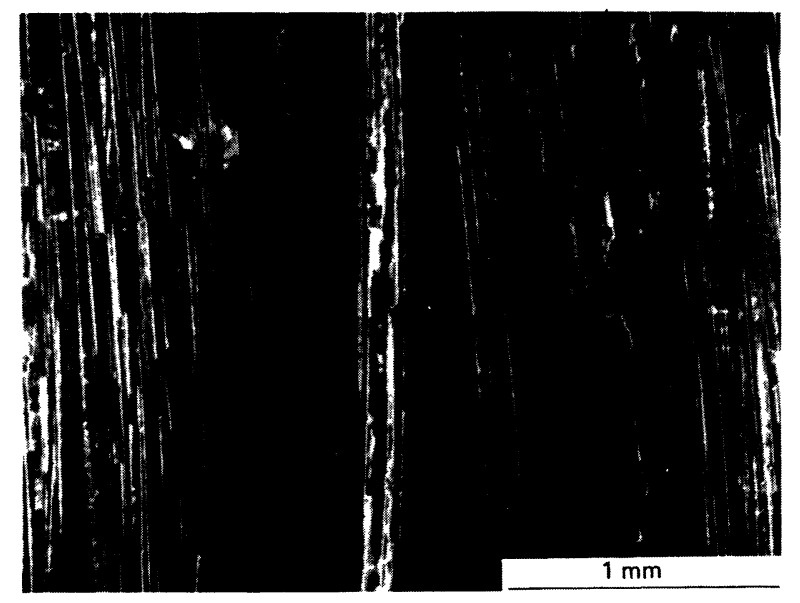

Fig. 10. - Etat de fissuration d'un matériau composite unidirectionel $\mathrm{SiC} /$ alumine après élaboration.

[Multiple fracture in as-elaborated SiC/alumina composite.] densification à température ambiante, d'un composite unidirectionnel $\mathrm{SiC} / \mathrm{Al}_{2} \mathrm{O}_{3}$ ayant un taux volumique de fibres d'environ $20 \%$.

Nous constatons une fissuration quasi régulière dans la zone à faible taux volumique de fibres $V_{\mathrm{f}}$, dont les espaces interfissures varient entre 58 et $150 \mu \mathrm{m}$. La zone à fort $V_{\mathrm{f}}$ donne des espaces interfissures entre 29 et $88 \mu \mathrm{m}$. La moyenne dans la surface observée se situe à $60 \mu \mathrm{m}$. Il apparaîtrait que la fissuration dans ce type de composite soit liée à la distribution locale des fibres. L'inhomogénéité observée est liée à la mise en place des torons (500 filaments) dans le moule d'infiltration. Cette observation de surface révèle qu'un certain nombre de fissures sont arrêtées par le renforcement.

6.2 EMISSION ACOUSTIQUE AU COURS DU PROCESSUS DE FRITTAGE. - L'évolution de cette microfissuration a été analysée expérimentalement par émission acoustique par l'équipe du Pr. Fantozzi au cours du traitement de frittage sur un composite unidirectionnel $\mathrm{SiC} / \mathrm{Al}_{2} \mathrm{O}_{3}$ non fritté de dimensions $20 \times$ $20 \times 3 \mathrm{~mm}^{3}$.

Durant la phase de chauffage jusqu'à $1200^{\circ} \mathrm{C}$ et de densification $\left(1 \mathrm{~h}\right.$ à $\left.1200^{\circ} \mathrm{C}\right)$, l'activité acoustique reste faible. Lors du refroidissement, par contre, le nombre de salves augmente fortement à partir de $800^{\circ} \mathrm{C}$, avec un maximum à $500^{\circ} \mathrm{C}$. En s'annulant vers $300{ }^{\circ} \mathrm{C}$, la fissuration atteint la saturation. La courbe de la figure 11 donne l'évolution du nombre de coups cumulés sur le nombre total de coups enregistrés au cours de l'activité acoustique en fonction de la température de refroidissement.

Des essais de traction réalisés à température ambiante après un tel traitement ont montré [4] que les fibres n'ont pas été endommagées (voir Chap. 6.4), et donc que l'émission acoustique est uniquement due à la fissuration matricielle.

6.3 ANALYSE MÉCANIQUE DE L'ÉMISSION ACOUSTIQUE. - A l'aide de l'algorithme décrit au chapitre 4, nous avons simulé l'évolution de la microfissuration matricielle d'un composite monofilamentaire $\mathrm{SiC} /$ alumine, au cours du refroidissement. Les données utilisées sont issues d'études précédentes sur les matériaux décrits au chapitre 6.1, à savoir :

- module d'Young de la matrice : $60 \mathrm{GPa}$

- contrainte moyenne de rupture de la matrice : $50 \mathrm{MPa}$

- porosité : $35 \%$

- seuil de glissement $\tau_{1}$ mesuré à température ambiante par microindentation : $37-64 \mathrm{MPa}$

- longueur du composite : $20 \mathrm{~mm}$

- taux volumique de fibres : $20 \%$

- module d'Young de la fibre $\mathrm{SiC}$ Nicalon : $200 \mathrm{GPa}$

- diamètre moyen de la fibre : $15 \mu \mathrm{m}$.

La valeur du module de Weibull a été prise égale à 


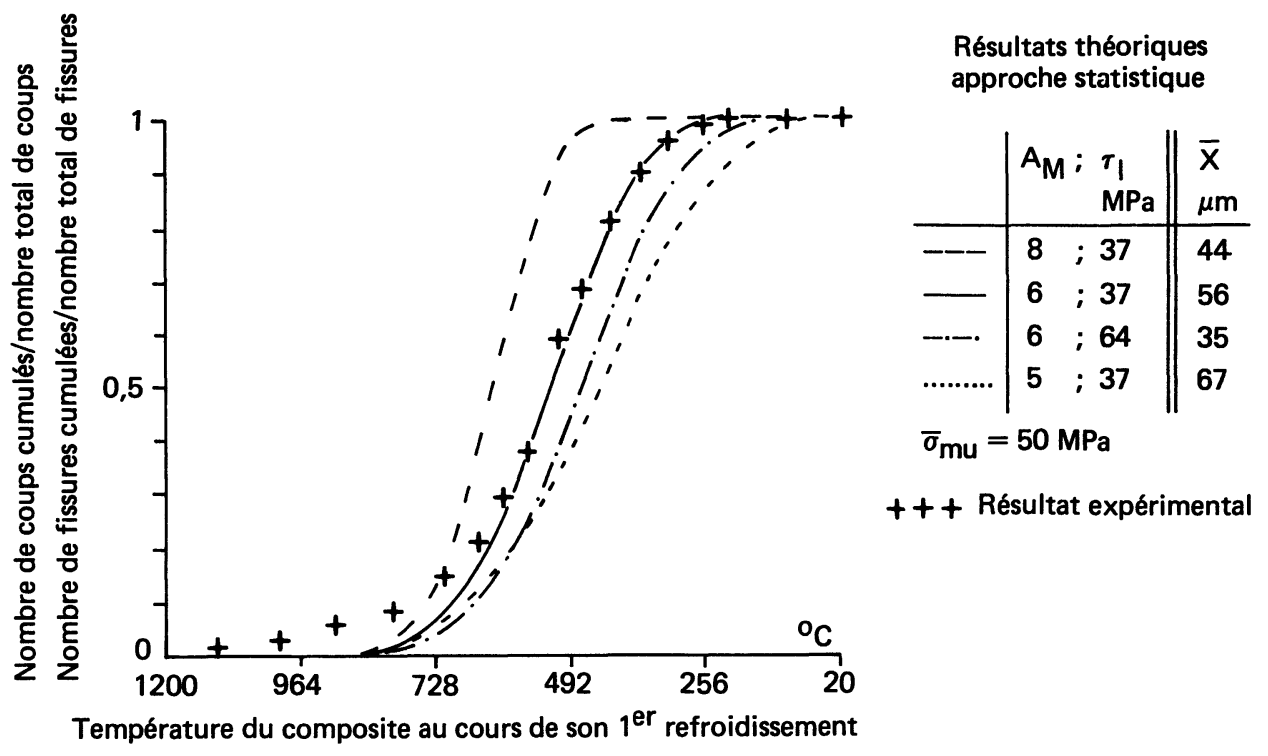

Fig. 11. - Evolution de la microfissuration d'origine thermomécanique en fonction du refroidissement dans un composite SiC/alumine unidirectionnel. +++ : émission acoustique, --_ : émission numérique du refroidissement d'un composite monofilamentaire analogue : approche statistique de la rupture.

[Thermomechanical microcracking evolution in an unidirectional SiC/alumina composite during cooling. $+++:$ acoustic emission, -- : : computed curve.]

6 (valeur parfaitement plausible), donnant, pour la contrainte moyenne de rupture de $50 \mathrm{MPa}$, le paramètre d'échelle de la loi de Weibull de $25,7 \mathrm{MPa} . \mathrm{mm}^{3 / 6}$ pour un volume de référence de matrice équivalent à un $V_{\mathrm{m}}$ de $80 \%$.

La simulation décrit (Fig. 11) une progression de la fissuration au cours du refroidissement qui débute vers $800^{\circ} \mathrm{C}$ et qui atteint la saturation vers $300^{\circ} \mathrm{C}$. Nous avons porté l'évolution du nombre de fissures cumulées rapporté au nombre total de fissures en fonction de la température du premier refroidissement.

Si cette simulation ne prétend pas représenter la réalité globale du comportement d'un tel composite multifilamentaire, elle dégage néanmoins une première analogie suffisante pour poursuivre une analyse de ce phénomène non linéaire. Une analyse de la sensibilité du module de Weibull (paramètre non déterminé expérimentalement) montre que ce paramètre est susceptible de modifier très fortement l'allure des courbes en S. Plus $a_{\mathrm{m}}$ est grand, plus le domaine de température au cours duquel s'effectuent ces microfissurations est réduit. Par contre, $\tau_{1}$ ne modifie pas leur allure générale mais opère une quasi-translation des courbes. Quand $\tau_{1}$ augmente, le domaine de température s'élargit, induisant ainsi un retard à la saturation de la microfissuration et une diminution de la distance moyenne interfissure, $\bar{x}$ (Tab. de la Fig. 11).
6.4 COMPORTEMENT EXPÉRIMENTAL EN CHARGEMENT CYCLIQUE. - Des essais de traction avec cyclage ont été entrepris sur ces composites unidirectionnels densifiés de dimensions $2 \times 2 \times 60 \mathrm{~mm}^{3}$, possédant un taux volumique de fibres d'environ $25 \%$.

Les courbes de traction $\sigma / \varepsilon$ ont été obtenues avec un extensomètre optique pour une longueur de jauge de $26 \mathrm{~mm}$ (Fig. 12). Ces éprouvettes préfissurées présentent au début du chargement des modules d'élasticité supérieurs à la valeur $E_{\mathrm{f}} V_{\mathrm{f}}(\approx 50 \mathrm{GPa})$.

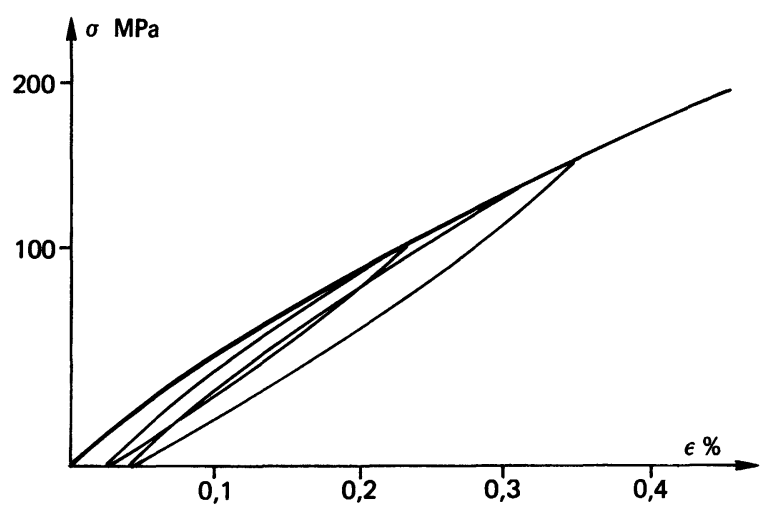

Fig. 12. - Essai de traction alternée sur composite unidirectionnel SiC/alumine.

[Cycling tensile test on an unidirectional SiC/alumina composite.] 
Ceci s'explique par le fait que la matrice, même fissurée, contribue à la rigidité du composite. Au fur et à mesure de son allongement, les glissements à l'interface abaissent la rigidité apparente du composite jusqu'à une valeur limite de $E_{\mathrm{f}} V_{\mathrm{f}}$, atteinte ici à partir de $0,1 \%$ de déformation moyenne. Au-delà de $0,3 \%$ de déformation, des ruptures de fibres interviennent, faisant chuter le module d'élasticité. Dans le domaine des déformations (jusqu'à $0,3 \%$ ), où la potentialité des fibres a été maintenue, deux cyclages ont été réalisés.

Les pentes de chargement et de déchargement sont quasi identiques, de l'ordre de $65 \mathrm{GPa}$. Cette valeur est proche de celle de la pente à l'origine $(64 \mathrm{GPa})$. Les boucles d'hystérésis présentent dans leur ensemble la forme des boucles de frottement issues des simulations (Fig. 9a).

6.5 ANALYSE MÉCANIQUE DU CHARGEMENT CYCLIQUE. - La simulation d'un cyclage en traction sur composite monofilamentaire $\mathrm{SiC} /$ alumine ayant les mêmes caractéristiques que précédemment (Chap. 6.3) a permis de calculer les énergies de frottement des différentes boucles pour des seuils de glissement fixés à 37 et $64 \mathrm{MPa}$ (Fig. 13).

Faisant l'hypothèse que l'énergie dissipée dans la boucle d'hystérésis lors du cyclage en traction du composite multifilamentaire est égale à la somme des énergies élémentaires développées par chaque

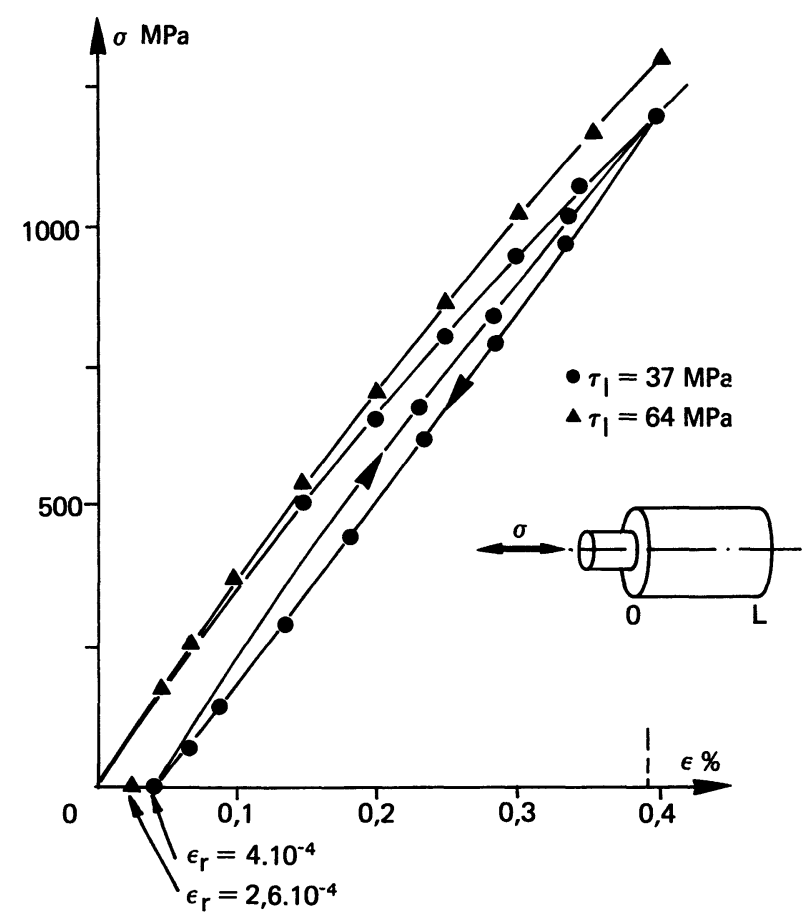

Fig. 13. - Simulation d'essai de traction alternée sur composite monofilamentaire SiC/alumine préfissuré $(L=0,1 \mathrm{~mm})$.

[Computed cycling tensile test on precracked SiC/alumina composite $(L=0.1 \mathrm{~mm})$.] fibre dans la zone d'influence de la fissure, nous pouvons écrire :

$$
W_{\mathrm{T}} \# 2 \cdot W_{\mathrm{D}} \cdot N_{\mathrm{f}} \cdot N
$$

où

$W_{\mathrm{T}}$ : énergie dissipée dans le composite multifilamentaire à une déformation moyenne donnée,

$W_{\mathrm{D}}$ : énergie dissipée dans le composite monofilamentaire à la même déformation moyenne que le composite multifilamentaire et au même taux volumique de fibres,

$N_{\mathrm{f}}$ : nombre de fissures dans le composite,

$N$ : nombre de fibres travaillant en frottement.

Des observations de traction de ces éprouvettes sous microscope électronique à balayage (Fig. 14) révèlent que toutes les fissures d'origine thermomécanique ne s'écartent pas lors du chargement. La distance moyenne entre les fissures travaillantes est de l'ordre de $200 \mu \mathrm{m}$. Une telle constatation pourrait être expliquée par des seuils de contraintes de cisaillement à l'interface $\left(\tau_{1}\right)$ différents, donnant ainsi la possibilité aux lèvres des fissures où les $\tau_{1}$ sont les plus faibles, de s'écarter fortement.

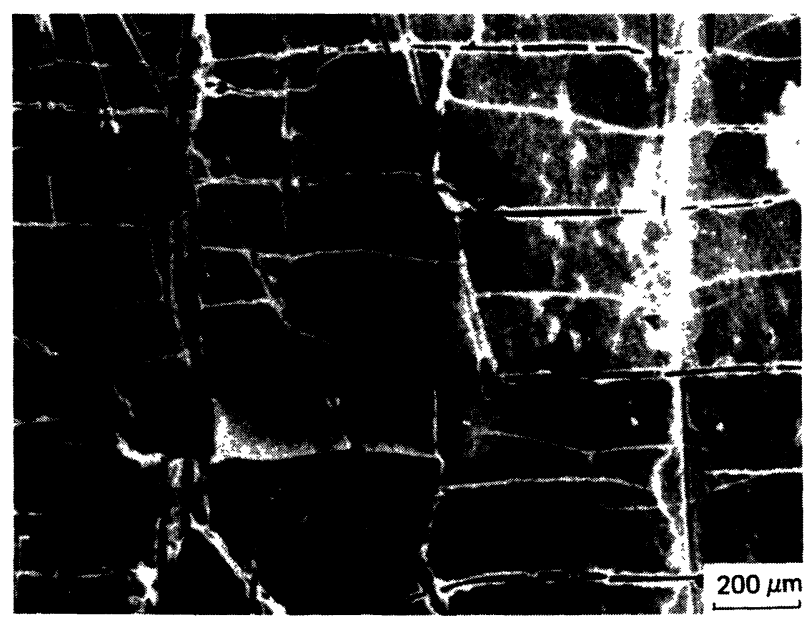

Fig. 14. - Micrographie d'un composite SiC/alumine sous charge (100 daN) lors d'un essai de traction sous microscope électronique à balayage.

[Scanning electron micrograph showing a SiC/alumina composite loaded in tension (100 daN).]

Considérant que ces fissures traversent toute la section du composite $\left(N_{\mathrm{f}}=130\right)$ et que l'ensemble des fibres travaillent $(N=5500)$, nous pouvons estimer les énergies mises en jeu lors des cyclages. Le tableau I récapitule les résultats expérimentaux et les valeurs théoriques pour les deux boucles.

Les énergies calculées sont du même ordre de grandeur que celles mesurées sur le composite unidirectionnel à déformations comparables. Nous notons que le rapport de déformation lors du 
Tableau I. - Comparaison entre expérience et simulation des énergies dissipées lors d'un cyclage en traction. Cas des composites SiC/alumine.

[Comparison of experimental results and numerical modelling on the energies dissipated in loading/unloading cycles in SiC/alumina composites.]

\begin{tabular}{|c|c|c|c|c|c|}
\hline $\begin{array}{c}\varepsilon \\
(\%)\end{array}$ & $\begin{array}{c}\tau_{1} \\
(\mathrm{MPa})\end{array}$ & $\begin{array}{c}l \\
(\mu \mathrm{m})\end{array}$ & $\begin{array}{c}W_{\mathrm{D}} \\
\left(\times 10^{-10}\right.\end{array}$ & $\begin{array}{c}W_{\mathrm{T}} \\
\left(\times 10^{-5} \mathrm{~J}\right)\end{array}$ & $\begin{array}{c}W_{\text {exp }} \\
\left(\times 10^{-5} \mathrm{~J}\right)\end{array}$ \\
\hline 0,23 & 37 & 36 & 7 & 100 & 112 \\
\hline 0,35 & 37 & 50 & 20 & 286 & 21 \\
\hline 64 & 32 & 15 & 214 & \\
\hline
\end{tabular}

déchargement $(\varepsilon \approx 0,4 \%)$ sur la déformation lors $\mathrm{du}$ rechargement est respectivement de 10 pour $\tau_{1}=37 \mathrm{MPa}$ et 15 pour $\tau_{1}=64 \mathrm{MPa}$, alors qu'expérimentalement on obtient à une même déformation un rapport de 10 (Fig. 13). Les longueurs de glissement $(l)$ calculées (Eq. (6)) valident par ailleurs l'observation microscopique dans la mesure où $l$ est très inférieur à la distance entre les fissures travaillantes.

Ainsi donc, il semblerait que les fissures travaillantes au cours de la traction ne concernent pas la totalité des fissures initiées au cours du premier refroidissement de ce premier matériau. Par conséquent, la notion de seuil de glissement unique ne semble pas pouvoir être retenue, et de plus cette valeur de $\tau_{1}$ peut évoluer au cours du déplacement. Cette approche pourra être étudiée expérimentalement à l'aide d'un dispositif d'indentation instrumentée, permettant de caractériser la loi de comportement interfacial lors d'un chargement monotone et cyclique.

\section{Conclusion.}

Ainsi, avec deux notions relativement simples communément admises :

- notion de frottement interfacial,

- notion de défauts,

ces modèles, décrits ci-dessus, permettent de décrire les mécanismes simples de la rupture de systèmes composites unidirectionnels céramique/céramique. L'apparition d'une fissure matricielle induit une singularité de contrainte de cisaillement à l'interface fibre-matrice qui, dans de tels systèmes, ne peut être absorbée qu'en supposant un glissement relatif entre les deux composants. Ce mécanisme fondamental de la rupture matricielle, associé à la notion de défauts et par suite de statistique de rupture, a permis de mettre en évidence des phénomènes que l'analyse linéaire n'explique pas :

1) La multifissuration apparaît à des allongements généralement supérieurs à ceux mesurés sur la matrice en absence de fibres.

2) Elle se développe généralement dans un domaine de contraintes relativement étendu.

3) La distance entre fissures observe une borne inférieure caractéristique du système fibre-matrice étudié.

4) Le composite possède un comportement non linéaire dissipatif conduisant à des cycles d'hystérésis.

Cette première approche, qui décrit la cinétique d'initiation de fissuration, nécessite cependant un approfondissement de la notion de volume matriciel. Elle ne représente pas la propagation de fissures faisant intervenir des notions de concentration de contraintes ou d'énergie de création de surface compte tenu du système élémentaire choisi. Par contre, l'analyse du système multifilamentaire sera regardé sous l'angle de la propagation d'une fissure perpendiculaire au renforcement, permettant ainsi de calculer les énergies dissipées. Une telle démarche nécessite de préciser la rhéologie de l'interface, traitée par ailleurs à l'aide de la méthode d'indentation instrumentée.

Cet article résume ainsi les premiers résultats d'une recherche en cours.

\section{Annexe A}

Les états de contrainte lors d'une fissuration de la matrice et après glissement à $\tau_{1}$ constant de la fibre par rapport à la matrice peuvent être modélisés en axisymétrique, comme suit (Fig. 1A).

La longueur de glissement à $\tau_{1}$ constant, $l$; vérifie l'équation suivante :

$$
l=\frac{1-V_{\mathrm{f}}}{V_{\mathrm{f}}} \cdot \frac{\sigma_{\mathrm{asy}}^{\mathrm{m}} \cdot r_{\mathrm{f}}}{2 \tau_{1}}
$$

avec

$\sigma_{\text {asy }}^{\mathrm{m}}$ contrainte de traction dans la matrice loin de la fissure $(z \geqslant l)$

$\sigma_{\text {app }}^{f}$ contrainte de traction dans la fibre au droit de la fissure

$\sigma_{\text {asy }}^{\mathrm{f}}$ contrainte de traction dans la fibre loin de la fissure $(z \geqslant l)$.

\section{Ecriture des équations.}

Pour $0 \leqslant z \leqslant l$ :

$$
\begin{array}{ll}
\sigma^{\mathrm{f}}(z)=\frac{-z}{l}\left(\sigma_{\text {app }}^{\mathrm{f}}-\sigma_{\text {asy }}^{\mathrm{f}}\right)+\sigma_{\text {app }}^{\mathrm{f}} & (\text { fibre }) \\
\sigma^{\mathrm{m}}(z)=\frac{z}{l} \sigma_{\text {asy }}^{\mathrm{m}} & \text { (matrice) }
\end{array}
$$




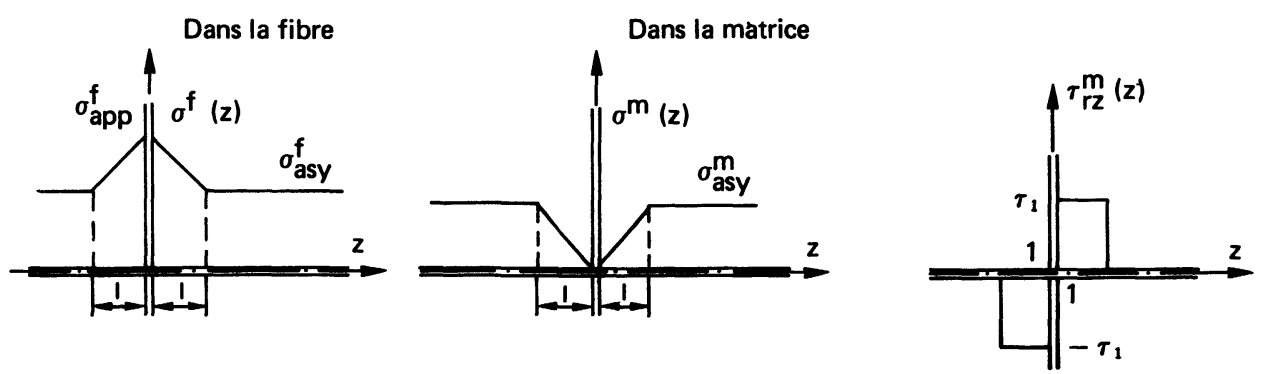

Fig. 1A. - Modélisation des états de contraintes dans le composite monofilamentaire après glissement interfacial.

[Stresses state in a single fibre composite after interfacial sliding.]

Pour $z \geqslant l$ :

$$
\begin{aligned}
& \sigma^{\mathrm{f}}(z)=\sigma_{\text {asy }}^{\mathrm{f}} \\
& \sigma^{\mathrm{m}}(z)=\sigma_{\text {asy }}^{\mathrm{m}} .
\end{aligned}
$$

En dehors de la zone de glissement, il y a égalité des déformations $(\varepsilon)$ :

$$
\varepsilon_{\mathrm{f}}=\varepsilon_{\mathrm{m}}
$$

d'où

$$
\frac{\sigma_{\text {app }}^{\mathrm{f}}}{\sigma_{\text {asy }}^{\mathrm{m}}}=\left(1+\frac{1}{\beta}\right) \frac{V_{\mathrm{m}}}{V_{\mathrm{f}}} \text { et } \frac{\sigma_{\text {app }}^{\mathrm{f}}}{\sigma_{\text {asy }}^{\mathrm{f}}}=1+\beta
$$

$\operatorname{avec} \beta=\frac{E_{\mathrm{m}}}{E_{\mathrm{f}}} \frac{V_{\mathrm{m}}}{V_{\mathrm{f}}}$

STATISTIQUE DE RUPTURE DANS LA MATRICE. Considérant des défauts de volume, la probabilité de rupture de la matrice s'écrit, pour une loi de Weibull à deux paramètres :

$$
P_{\mathrm{r}}\left(\sigma^{\mathrm{m}}(z)\right)=1-\exp \left[-\int_{V}\left(\frac{\sigma^{\mathrm{m}}(z)}{\sigma_{\mathrm{m}}^{\circ}}\right)^{a_{\mathrm{m}}} \mathrm{d} v\right]
$$

avec $\mathrm{d} v=2 \pi r \mathrm{~d} r \mathrm{~d} z$.

Domaine d'intégration et de probabilité de rupture.

Il concerne la distance entre deux fissures $(2 L)$.

\section{Intégration.}

D'après $(2)$ on $a$ :

$$
\begin{aligned}
P_{\mathrm{r}}\left(\sigma^{\mathrm{m}}(z)\right)=1-\exp \left[-\left(\int_{0}^{l}\left(\frac{\sigma^{\mathrm{m}}(z)}{\sigma_{\mathrm{m}}}\right)^{a_{\mathrm{m}}}+\right.\right. \\
\left.\left.+\int_{l}^{L}\left(\frac{\sigma^{\mathrm{m}}(z)}{\dot{\sigma}_{\mathrm{m}}}\right)^{a_{\mathrm{m}}}\right) \cdot 2 \cdot S_{\mathrm{m}} \cdot \mathrm{d} z\right]
\end{aligned}
$$

avec $S_{\mathrm{m}}=\pi\left(r_{\mathrm{m}}^{2}-r_{\mathrm{f}}^{2}\right)$.

Le coefficient 2 rend compte de la symétrie des contraintes dans le domaine $2 L$, d'où :

$$
\begin{aligned}
P_{\mathrm{r}}\left(\sigma^{\mathrm{m}}(z)\right)=1-\exp [ & -S_{\mathrm{m}} \cdot 2 L \cdot\left(\frac{\sigma_{\text {asy }}^{\mathrm{m}}}{\dot{\sigma}_{\mathrm{m}}}\right)^{a_{\mathrm{m}}} \times \\
& \left.\times\left(1+\frac{l}{L}\left(\frac{1}{a_{\mathrm{m}}+1}-1\right)\right)\right]
\end{aligned}
$$

et, compte tenu de l'hypothèse de rupture à $50 \%$ :

$$
P_{\mathrm{r}}\left(\sigma_{\mathrm{m}}(z)\right)=0,5=1-\exp (\ldots)
$$

on obtient :

$$
\sigma_{\text {asy }}=\stackrel{\circ}{\sigma}_{\mathrm{m}} \cdot\left(\frac{\ln 2}{2 L \cdot S_{\mathrm{m}}} \cdot \frac{1}{A}\right)^{1 / a_{\mathrm{m}}} .
$$

avec $A=1+\frac{l}{L}\left(\frac{1}{a_{\mathrm{m}}+1}-1\right)$.

Apparition de la première fissure dans la matrice.

$A$ traduit la contribution non uniforme du chargement de la matrice. Dans le cas où $L$ est grand par rapport à $l$, on a :

$A \approx 1$, traduisant ainsi un chargement uniforme .

L'apparition de la première fissure dans la matrice de longueur $2 L=2 L_{0}$ (où $2 L_{0}$ correspond à la longueur du composite considéré) s'opère à la contrainte $\sigma_{\text {asy }}^{\mathrm{m}}$ dans la matrice (d'après (A.4)) :

$$
\sigma_{\text {asy }}^{\mathrm{m}}=\stackrel{\circ}{\mathrm{m}}_{\mathrm{m}} \cdot\left(\frac{\ln 2}{2 L_{0} \cdot S_{\mathrm{m}}}\right)^{1 / a_{\mathrm{m}}}
$$

par un changement de variable, la contrainte équivalente appliquée au composite $\left(\sigma_{c}\right)$ s'exprime, d'après (A.3) :

$$
\begin{aligned}
\sigma_{\mathrm{c}}=\left(1+\frac{1}{\beta}\right) \cdot V_{\mathrm{m}} \cdot \stackrel{\circ}{\mathrm{m}}_{\mathrm{m}} \cdot\left(\frac{\ln 2}{2 L_{0} \cdot S_{\mathrm{m}}}\right)^{1 / a_{\mathrm{m}}} & \left(\sigma_{\mathrm{c}}=F / S\right) .
\end{aligned}
$$

Fissuration multiple de la matrice.

L'équation (4) lie la contrainte d'apparition de la 
fissure intercalaire à la distance interfissure $2 L$, nous permettant d'écrire :

$2 L=\frac{\ln 2}{S_{\mathrm{m}}} \cdot\left(\frac{\sigma_{\mathrm{m}}^{0}}{\sigma_{\mathrm{asy}}^{\mathrm{m}}}\right)^{a_{\mathrm{m}}}-2 l\left(\frac{1}{a_{\mathrm{m}}+1}-1\right)$
pour $L \geqslant l$

ou, par un changement de variable analogue $\left(\sigma_{\mathrm{c}}\right)$ :

$2 L=\frac{\ln 2}{S_{\mathrm{m}}} \cdot\left(\frac{\stackrel{\circ}{\mathrm{m}}_{\mathrm{m}} \cdot V_{\mathrm{m}} \cdot(1+1 / \beta)}{\sigma_{\mathrm{c}}}\right)^{a_{\mathrm{m}}}-$ $-2 l\left(\frac{1}{a_{\mathrm{m}}+1}-1\right)$.

Cas de la saturation.

La condition $L$ calculée, inférieure ou égale à la longueur de glissement entre la fibre et la matrice (l), déterminée à partir de l'équation (1), définit la saturation.

Sur toute la longueur $2 L$, il y a glissement et les contraintes matricielles restent constantes lors de chargements supplémentaires.

$$
\begin{aligned}
\sigma^{\mathrm{m}}(z)=z /\left(\frac{1-V_{\mathrm{f}}}{V_{\mathrm{f}}} \cdot \frac{r_{\mathrm{f}}}{2 \tau_{1}}\right) ; \\
\sigma^{\mathrm{f}}(z)=\sigma_{\text {app }}^{\mathrm{f}}-\frac{z}{r_{\mathrm{f}}} \cdot 2 \tau_{1} .
\end{aligned}
$$

D'après (A.4), il vient :

$$
A=\frac{1}{a_{\mathrm{m}}+1} \quad \text { pour } \quad l=L .
$$

STATISTIQUE DE RUPTURE DANS LA FIBRE. - Considérant les défauts par unité de longueur, la probabilité de rupture de la fibre pour une loi de Weibull à deux paramètres s'écrit :

$$
P_{\mathrm{r}}\left(\sigma^{\mathrm{f}}(z)\right)=1-\exp \left(-\int_{v}\left(\frac{\sigma^{\mathrm{f}}(z)}{\stackrel{\sigma}{\mathrm{f}}_{\mathrm{f}}}\right)^{a_{\mathrm{f}}} \mathrm{d} v\right)
$$

avec $\mathrm{d} v=S_{\mathrm{f}} \mathrm{d} z$ et $S_{\mathrm{f}}=\Pi r_{\mathrm{f}}^{2}$.

Domaine d'intégration et de probabilité de rupture.

Le domaine d'intégration concerne la distance entre deux fissures existantes $(2 L)$ (voir Fig. 2A).

De façon analogue à l'étude de la matrice, on choisira la probabilité de rupture de la fibre à $50 \%$. Cependant, sa rupture est concernée par l'ensemble des fissurations de la matrice sur toute la longueur du composite $\left(2 L_{0}\right)$, et donc nous pouvons écrire que :

$$
\begin{aligned}
P_{\mathrm{r}}\left(\sigma^{\mathrm{f}}(z)\right)=1 & -\exp \left[-\left(\int_{0}^{l}\left(\frac{\sigma^{\mathrm{f}}(z)}{\sigma_{\mathrm{f}}}\right)\right)^{a_{\mathrm{f}}}+\right. \\
& \left.+\int_{l}^{L}\left(\frac{\sigma^{\mathrm{f}}(z)}{\sigma_{\mathrm{f}}}\right)^{a_{\mathrm{f}}} \cdot 2 \cdot S_{\mathrm{f}} \cdot L_{0} / L \cdot \mathrm{d}_{z}\right]
\end{aligned}
$$

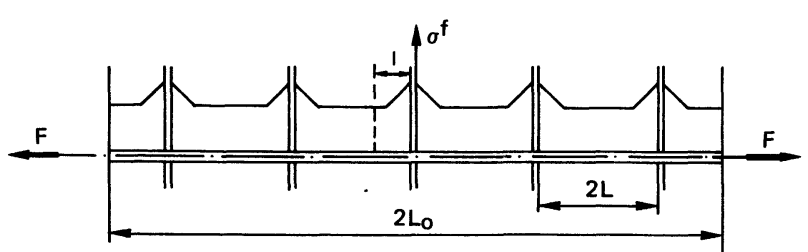

Fig. 2A. - Modélisation de la rupture du renfort dans un composite monofilamentaire.

[Fibre cracking in a single fibre composite.]

où $L_{0} / L$ rend compte en première approximation de la rupture sur la longueur totale du composite (2 $\left.L_{0}\right)$.

\section{Intégration.}

D'après (A.6), on obtient après intégration :

$$
P_{\mathrm{r}}\left(\sigma^{\mathrm{f}}(z)\right)=1-\exp \left[-2 L_{0} \cdot S_{\mathrm{f}} \cdot\left(\frac{\sigma_{\text {asy }}^{\mathrm{f}}}{\stackrel{\sigma}{\mathrm{f}}_{\mathrm{f}}}\right)^{a_{\mathrm{f}}} \cdot B\right]
$$

avec

$$
B=\frac{l}{L} \cdot \frac{1}{a_{\mathrm{f}}+1} \cdot \frac{1}{\beta}\left[(1+\beta)^{a_{\mathrm{f}}+1}-1\right]+(1-l / L)
$$

d'où, à $50 \%$ de probabilité de rupture :

$$
P_{\mathrm{r}}\left(\sigma^{\mathrm{f}}(z)\right)=0,5=1-\exp (\ldots)
$$

il vient :

$$
\sigma_{\text {asy }}^{\mathrm{f}}=\stackrel{\circ}{\sigma}_{f}\left(\frac{\ln 2}{S_{\mathrm{f}} \cdot 2 L_{0}} \cdot \frac{1}{B}\right)^{1 / a_{\mathrm{f}}}
$$

où, compte tenu du changement de variable $\left(\sigma_{\mathrm{c}}\right)$ :

$$
\sigma_{\mathrm{c}}=(1+\beta) V_{\mathrm{f}} \cdot \stackrel{\circ}{\sigma}_{\mathrm{f}}\left(\frac{\ln 2}{S_{\mathrm{f}} \cdot 2 L_{0}} \cdot \frac{1}{B}\right)^{1 / a_{\mathrm{f}}}
$$

- à l'apparition de la première fissure dans la matrice, on a comme précédemment : $B=1$;

— à la saturation de la fissuration dans la matrice :

$$
B=\frac{1}{a_{\mathrm{f}}+1} \cdot \frac{1}{\beta}\left[(1+\beta)^{a_{\mathrm{f}}+1}-1\right] .
$$

COURBES CONTRAINTES DÉFORMATIONS. - Lors de la fissuration de la matrice, on calcule la déformation moyenne de la fibre, $\bar{\varepsilon}_{\mathrm{f}}$, entre deux fissures distantes de $2 L$.

En effet, cette déformation moyenne est équivalente à celle qui serait calculée sur le composite entier, d'où :

$$
\bar{\varepsilon}_{\mathrm{f}}=\frac{1}{L}\left[\int_{0}^{l} \frac{\sigma^{\mathrm{f}}(z)}{E_{f}} \mathrm{~d} z+\int_{l}^{L} \frac{\sigma^{\mathrm{f}}(z)}{E_{\mathrm{f}}} \mathrm{d} z\right] .
$$


Par substitution des équations (A.6) et par intégration, on obtient :

$$
\bar{\varepsilon}_{\mathrm{f}}=\frac{\sigma_{\mathrm{c}}}{E_{\mathrm{c}}}\left[1+\frac{l}{2 L} \cdot \frac{E_{\mathrm{m}}}{E_{\mathrm{f}}} \cdot\left(\frac{1-V_{\mathrm{f}}}{V_{\mathrm{f}}}\right)\right]
$$

avec

$$
\sigma_{\mathrm{c}}=\sigma_{\mathrm{app}}^{\mathrm{f}} \cdot V_{\mathrm{f}} .
$$

Quand la longueur de glissement $l$ vaut $L$, la déformation moyenne s'écrit :

$$
\bar{\varepsilon}_{\mathrm{f}}=\frac{\sigma_{\mathrm{c}}}{E_{\mathrm{f}} V_{\mathrm{f}}}\left(1-\frac{1}{2} \cdot \frac{E_{\mathrm{m}} V_{\mathrm{m}}}{E_{\mathrm{c}}}\right) .
$$

A la saturation de la fissuration, il n'y a plus d'évolution des contraintes dans la matrice puisqu'il y a glissement sur toute la longueur du composite $\left(2 L_{0}\right)$. Dans ces conditions, la déformation moyenne sur la longueur critique $l_{\mathrm{c}}\left(l_{\mathrm{c}}=l=L\right)$ s'écrit :

$\bar{\varepsilon}_{\mathrm{f}}=\frac{1}{E_{\mathrm{f}} l_{\mathrm{c}}} \int_{0}^{l_{\mathrm{c}}}\left(\sigma_{\text {app }}^{\mathrm{f}}-\frac{z}{r_{\mathrm{f}}} \cdot 2 \tau_{1}\right) \mathrm{d} z$

d'après (A.6)

d'où

$$
\bar{\varepsilon}_{\mathrm{f}}=\frac{\sigma_{\mathrm{c}}}{E_{\mathrm{f}} V_{\mathrm{f}}}-\frac{l_{\mathrm{c}} \cdot \tau_{1}}{r_{\mathrm{f}} \cdot E_{f}}
$$

La pente de la courbe de traction est constante et est égale à $E_{\mathrm{f}} V_{\mathrm{f}}$ avec un décalage à l'origine de :

$$
\frac{l_{\mathrm{c}} \cdot \tau_{1}}{r_{\mathrm{f}} \cdot E_{\mathrm{f}}}
$$

\section{Annexe B}

Les déplacements relatifs fibre-matrice le long de l'axe de traction calculés à partir du programme de différences finies (Chap. 3.2) lors du chargement et du déchargement sont illustrés par la figure suivante (Fig. 1B).

L'énergie dissipée dans la boucle d'hystérésis est proportionnelle à la surface à l'aire comprise entre les courbes 1 et 3 .

- Compte tenu des équations simplifiées des contraintes lors du glissement (Eq. 2.A), les déplacements interfaciaux au chargement s'écrivent :

$$
\begin{aligned}
& \Delta u^{\mathrm{I}}=D \cdot\left(z^{2}-l^{2}\right)+\frac{\sigma_{\text {app }}^{\mathrm{f}}}{E_{\mathrm{f}}}(l-z) \\
& 0 \leqslant z \leqslant 1
\end{aligned}
$$

avec

$$
D=\frac{\tau_{1} E_{\mathrm{c}}}{E_{\mathrm{f}} r_{\mathrm{f}} E_{\mathrm{m}} V_{\mathrm{m}}}
$$

Cette équation décrit bien la courbe 1.

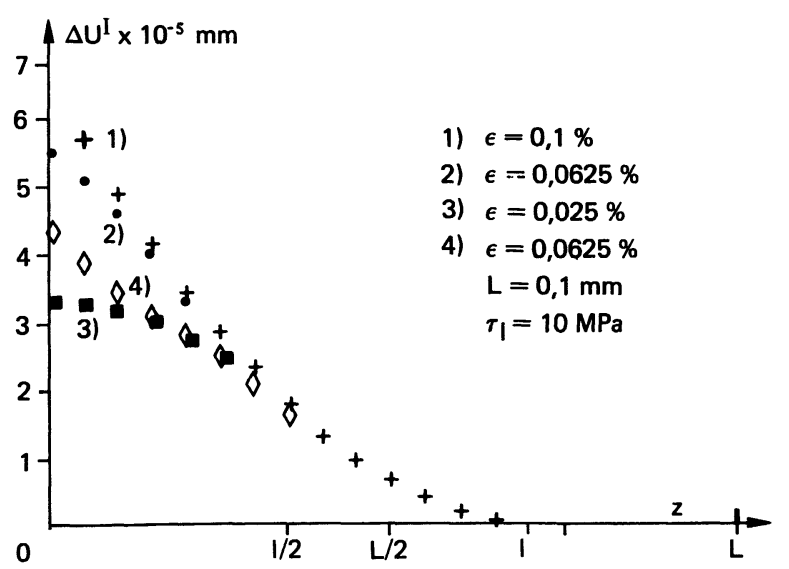

Fig. 1B. - Evolution des déplacements relatifs fibrematrice à l'interface le long de l'axe de traction au cours du chargement - Cas composite monofilamentaire SiC/verre.

[Fibre-matrix relative displacements along the tensile axis during loading, in a SiC/glass composite.]

- Au déchargement, les contraintes de traction dans la fibre et la matrice (Fig. 9b,c) peuvent être modélisées comme suit (Fig. 2B) :
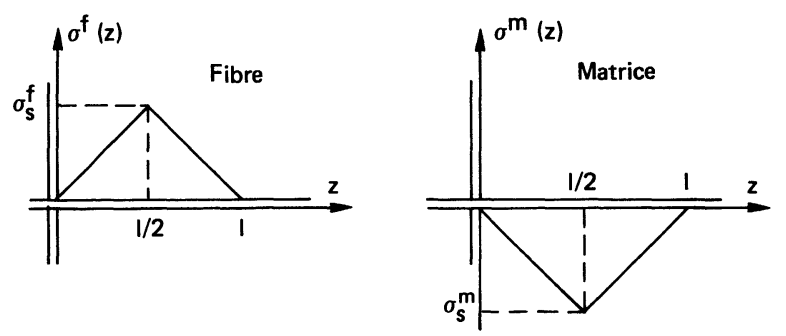

Fig. 2B. - Modélisation des états de contraintes dans le composite monofilamentaire après déchargement.

[Stresses state in a single fibre composite after unloading.]

avec

$$
\sigma_{\mathrm{s}}^{\mathrm{f}} \cdot V_{\mathrm{f}}+\sigma_{\mathrm{s}}^{\mathrm{m}} \cdot V_{\mathrm{m}}=0 .
$$

Les déplacements interfaciaux au déchargement découlant des états de contraintes simplifiés ci-dessus s'écrivent :

$$
\begin{aligned}
& \Delta u^{\mathrm{II}}=D .\left(l^{2} / 2-z^{2}\right) \quad 0 \leqslant z \leqslant l / 2 \\
& \Delta u^{\mathrm{II}}=D .(l-z)^{2} \quad l / 2 \leqslant z \leqslant l .
\end{aligned}
$$

Ces équations lissent bien la courbe 3 dans leurs domaines d'application respectifs.

La surface comprise entre les courbes 1 et 3 correspondant à un demi-cycle s'écrit donc :

$$
\Delta u=\int_{0}^{l / 2}\left(\Delta u^{\mathrm{I}}-\Delta u^{\mathrm{II}}\right) \mathrm{d} z+\int_{l / 2}^{l}\left(\Delta u^{\mathrm{I}}-\Delta u^{\mathrm{II}}\right) \mathrm{d} z
$$




$$
\begin{aligned}
\Delta u= & \int_{0}^{l / 2}\left[D\left(2 z^{2}-3 l^{2} / 2\right)\right. \\
& \left.-\sigma_{\text {app }}^{\mathrm{f}} / E_{\mathrm{f}}(z-1)\right] \mathrm{d} z \\
& +\int_{l / 2}^{l}\left(2 D l-\sigma_{\mathrm{app}}^{\mathrm{f}} / E_{\mathrm{f}}\right)(z-l) \mathrm{d} z \\
\Delta u= & \frac{\sigma_{\text {app }}^{\mathrm{f}}}{E_{\mathrm{f}}} \cdot \frac{l^{2}}{2}-\frac{22}{24} D l^{3}
\end{aligned}
$$

Dans le cas particulier où il y a liaison forte entre la fibre et la matrice au-delà de la zone de glissement, l'équation (B.3) s'écrit :

$$
\begin{aligned}
\Delta u=\frac{1}{96} \cdot \frac{1}{E_{\mathrm{f}}^{3} \cdot D^{2}} \cdot \sigma_{\mathrm{app}}^{\mathrm{f}^{3}} & \\
& \left(\text { sachant que } \sigma_{\mathrm{app}}^{\mathrm{f}}=2 E_{f} \cdot D \cdot l\right) .
\end{aligned}
$$

L'énergie dissipée dans la boucle d'hystérésis $W_{\mathrm{D}}$ s'exprime :

$$
W_{\mathrm{D}}=2 \pi r_{\mathrm{f}} \cdot(2 \cdot \Delta u) \cdot \tau_{1}
$$

c'est-à-dire

$$
W_{\mathrm{D}}=2 \pi r_{\mathrm{f}} l^{2} \frac{\tau_{1}}{E_{\mathrm{f}}}\left[\sigma_{\mathrm{app}}^{\mathrm{f}}-\frac{11}{6} \cdot \frac{E_{\mathrm{c}}}{E_{\mathrm{m}} V_{\mathrm{m}}} \cdot \frac{l}{r_{\mathrm{f}}} \cdot \tau_{1}\right]
$$

Dans le cas où la zone de glissement ne concerne pas la totalité de la longueur $L$, on a :

$$
W_{\mathrm{D}}=\frac{\pi}{24} \cdot\left(\frac{E_{\mathrm{m}} V_{\mathrm{m}}}{E_{\mathrm{c}}}\right)^{2} \cdot \frac{1}{E_{\mathrm{f}}} \cdot \frac{r_{f}^{3}}{\tau_{1}} \cdot \sigma_{\mathrm{app}}^{\mathrm{f}^{3}} .
$$

\section{Bibliographie}

[1] Aveston, J., Cooper, G. A. and Kelly, A., « Single and multiple fracture ", in The properties of fiber composites, p. 15-26. Conferences Proceedings of the National Physical Laboratory, (IPC Science and Technology Press Ltd, Surrey, England) 1971.

[2] Marshall, D. B., Cox, B. N. and Evans, A. G.,
The mechanics of matrix cracking in brittlematrix fiber composites, Acta Metall. 33 (1985) 2013-2021.

[3] Pérès, P., Jamet, J., ANouez, L., R. T. ONERA $n^{\circ}$ 19/3548 M, novembre 1986.

[4] Jamet, J., Ritti, M. H., Coyle, T. W., R. T. ONERA n $16 / 3548 \mathrm{M}$, février 1986. 